\title{
ARTICLE \\ A disintegrin and metalloproteinase domain-15 deficiency leads to exaggerated cigarette smoke-induced chronic obstructive pulmonary disease (COPD)-like disease in mice
}

\author{
Xiaoyun Wang ${ }^{1,2}$, Joselyn Rojas-Quintero ${ }^{1}$, Duo Zhang ${ }^{3,4}$, Takahiro Nakajima ${ }^{1}$, Katherine H. Walker ${ }^{1}$, Hong Yong Peh ${ }^{1,5}$, Yuhong Li ${ }^{1}$,
} Quynh-Anh Fucci ${ }^{1}$, Yohannes Tesfaigzi ${ }^{1}$ and Caroline A. Owen (iD) ${ }^{1}$

A disintegrin and metalloproteinase domain-15 (ADAM15) is expressed by cells implicated in the pathogenesis of chronic obstructive pulmonary disease (COPD), but its contributions to COPD are unknown. To address this gap, ADAM15 levels were measured in samples from cigarette smoke (CS)-versus air-exposed wild-type (WT) mice. CS-induced COPD-like disease was compared in CS-exposed WT, Adam $15^{-1-}$, and Adam 15 bone marrow chimeric mice. CS exposure increased Adam 15 expression in lung macrophages and $\mathrm{CD}^{+} \mathrm{T}$ cells and to a lesser extent in airway epithelial cells in WT mice. CS-exposed Adam $15^{-/-}$mice had greater emphysema, small airway fibrosis, and lung inflammation (macrophages and CD8 ${ }^{+} \mathrm{T}$ cells) than WT mice. Adam15 bone marrow chimera studies revealed that Adam 15 deficiency in leukocytes led to exaggerated pulmonary inflammation and COPD-like disease in mice. Adam 15 deficiency in $\mathrm{CD}^{+}$T cells was required for the exaggerated pulmonary inflammation and COPD-like disease in CS-exposed Adam $15^{-1-}$ mice (as assessed by genetically deleting CD8 ${ }^{+}$T cells in Adam $15^{-1-}$ mice). Adam 15 deficiency increased pulmonary inflammation by rendering $\mathrm{CD}^{+} \mathrm{T}$ cells and macrophages resistant to CS-induced activation of the mitochondrial apoptosis pathway by preserving mTOR signaling and intracellular Mcl-1 levels in these cells. These results strongly link ADAM15 deficiency to the pathogenesis of COPD.

Mucosal Immunology (2021) 14:342-356; https://doi.org/10.1038/s41385-020-0325-3

\section{INTRODUCTION}

COPD is the third leading cause of death worldwide. ${ }^{1}$ COPD is characterized by airflow limitation that is poorly reversible and associated with an abnormal pulmonary inflammatory response to noxious particles or gases including those contained in cigarette smoke (CS). ${ }^{2}$

Airflow limitation in COPD is caused both by pulmonary emphysema and small airway disease. ${ }^{3}$ Inhaling CS induces alveolar septal cell apoptosis ${ }^{4}$ and stimulates the recruitment of leukocytes into the lung. Proteinases released by CS-activated leukocytes and epithelial cells degrade extracellular matrix (ECM) proteins within the alveolar walls to promote emphysema development. $^{2}$

Prior studies of proteinases in COPD have focused on the activities of matrix metalloproteinases (MMPs) and serine and cysteine proteinases. ${ }^{2,5,6}$ Since the 1960 s, proteinases were thought to exclusively promote the development of COPD. ${ }^{6,7}$ However, this dogma was recently challenged when a disintegrin and metalloproteinase domain-8 (Adam8) was identified as the first proteinase that protects mice from developing CS-induced COPD-like disease. ${ }^{8}$ ADAMs are type I transmembrane proteinases with multiple domains, including (1) a pro-domain which maintains latency of the MP domain; (2) a MP domain which can shed proteins from cell surfaces; (3) disintegrin and cysteine-rich domains which can regulate cell adhesion and migration; (4) a surface-anchoring transmembrane domain; and (5) a cytoplasmic domain which can regulate intracellular signaling. ${ }^{9-11}$ Whether other ADAMs regulate CS-induced COPD-like disease in mice is unclear.

ADAM15 has potential to contribute to the pathogenesis of COPD. Human ADAM15 and murine Adam15 are expressed by cells implicated in the pathogenesis of COPD including leukocytes, epithelial cells, and fibroblasts. ${ }^{2}$ Although ADAM15 has minimal ECM protein-degrading activity, ${ }^{12}$ it has activities that could be relevant to the pathogenesis of COPD including shedding growth factor receptors, ${ }^{13}$ and regulating cellular adhesion and migration. $^{12-14}$ However, ADAM15's contributions to COPD have not been explored. We quantified and localized ADAM 15 expression in the lungs of CS- versus air-exposed WT mice and compared COPDlike lung disease in CS-exposed WT versus Adam $15^{-1-}$ mice and Adam 15 bone marrow (BM) chimeric mice. Our results show that Adam 15 deficiency promotes exaggerated COPD-like disease in CS-exposed mice by inciting an excessive pulmonary inflammatory response by increasing the survival of $\mathrm{CD}^{+} \mathrm{T}$ cells and macrophages by inhibiting CS-induced activation of the mitochondrial apoptosis pathway by abrogating CS-induced inactivation of mTOR-Mcl-1 signaling in these cells.

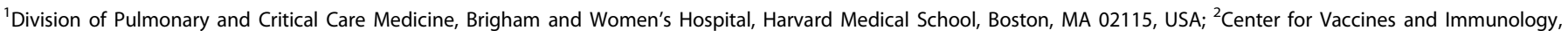

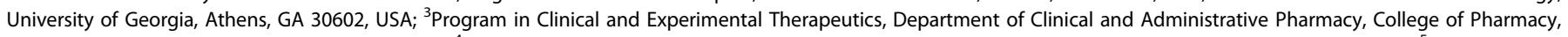

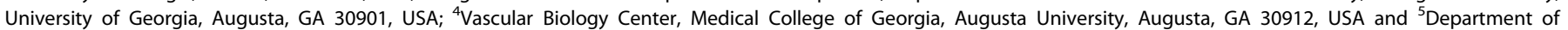
Pharmacology, Yong Loo Lin School of Medicine, National University Health System, Singapore, Singapore

Correspondence: Caroline A. Owen (cowenusa@yahoo.com) 

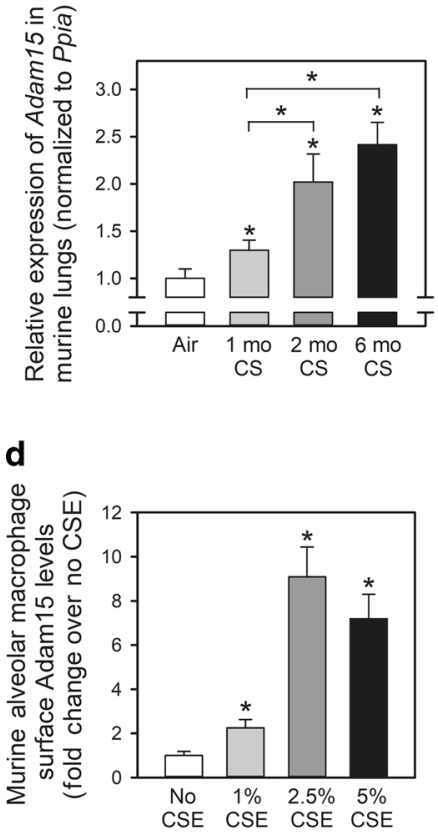

g

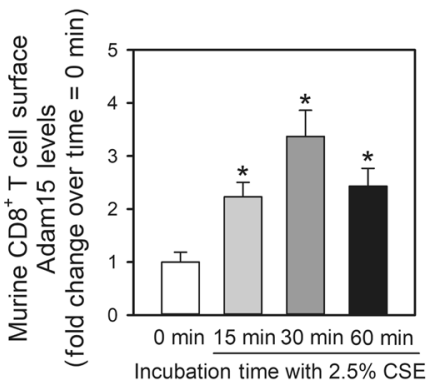

b

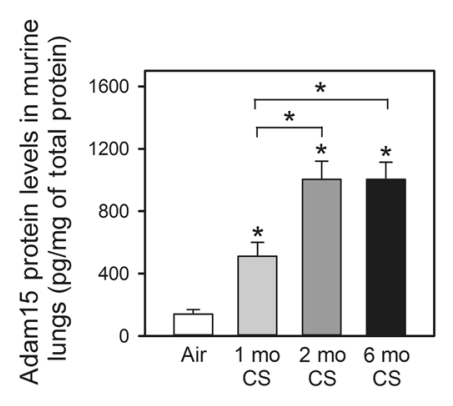

e

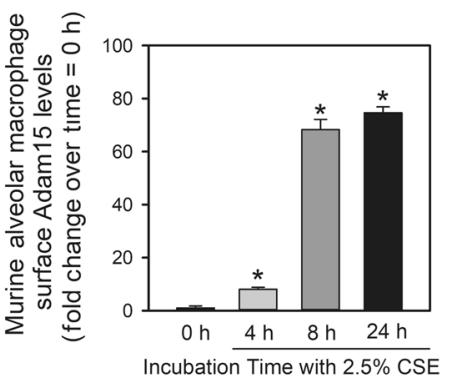

h

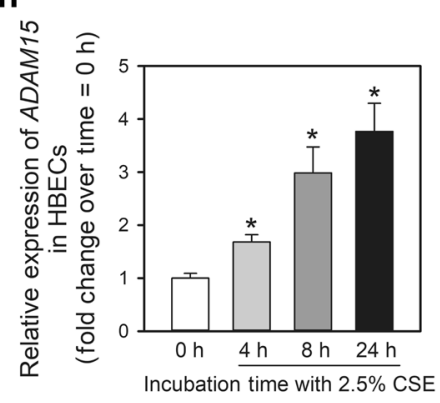

C

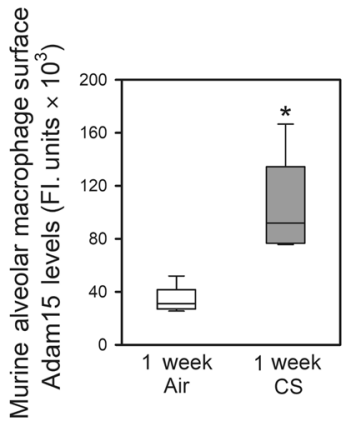

f

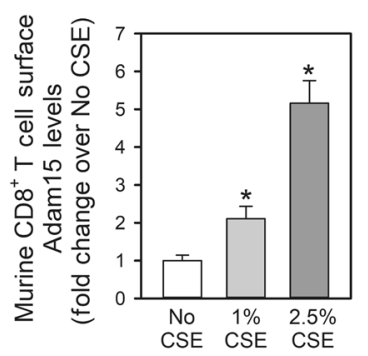

i

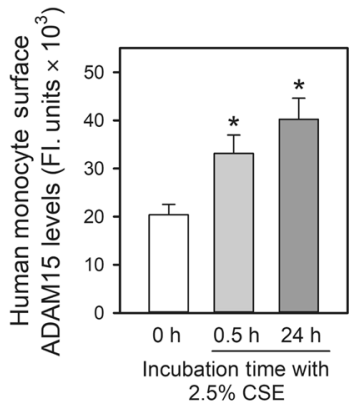

j

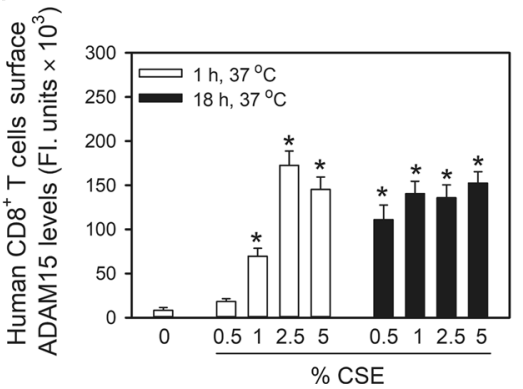

\section{RESULTS}

Adam15 expression is increased in the lungs of CS-exposed WT mice

Exposing WT mice to CS for 1 month increased lung Adam15 transcript and protein levels (Fig. 1a, b); levels increased further after 2 months of CS exposure but did not increase further after 6 months of CS exposure. Adam15 immuno-staining was detected in alveolar macrophages (AMs), $\mathrm{CD}^{+} \mathrm{T}$ cells, bronchial and alveolar epithelial cells, and a-smooth muscle actin (a-SMA)positive cells around the small airways in WT mice that were exposed to CS for 6 months (Fig. E1).
Surface Adam15 staining levels were measured on AMs isolated from WT mice that were exposed to air or CS for 1 week. Acute CS exposure increased surface Adam 15 levels on AMs approximately threefold (Fig. 1c). CS extract (CSE)-induced concentration- and time-dependent increases in surface Adam15 protein levels on AMs isolated from unchallenged WT mice (Fig. $1 \mathrm{~d}, \mathrm{e}$ ). $\mathrm{CD}{ }^{+}{ }^{+} \mathrm{T}$ cells are increased in the lungs of CS-exposed mice and COPD patients and are required for CS-induced lung inflammation and airspace enlargement in mice. ${ }^{15-17}$ We investigated whether CSE regulates surface Adam 15 levels on $\mathrm{CD}^{+} \mathrm{T}$ cells. CSE induced concentration-dependent increases in surface Adam15 levels on 
344

Fig. 1 A disintegrin and a metalloproteinase domain 15 (Adam15) expression is induced in the lungs of wild-type (WT) mice exposed to cigarette smoke (CS) and is upregulated on the surface of macrophages, monocytes and CD8 ${ }^{+} \mathbf{T}$ cells exposed to CS extract (CSE) in vitro. a, b Adam15 steady state mRNA and protein levels were measured in the lungs of C57BL/6 WT mice that were exposed to air or CS for up to 6 months using quantitative real-time PCR and an ELISA, respectively ( $n=9-10$ mice/group). The bar graphs show means \pm SD. Data were analyzed using one-way ANOVAs followed by pair-wise testing with two-tailed Student's $t$ tests. ${ }^{*} P \leq 0.004$ versus air-exposed mice belonging to the same genotype control or the group indicated. c C57BL/6 WT mice were exposed to air or CS for 1 week, and alveolar macrophages (AMs) were isolated using bronchoalveolar lavage. Surface Adam 15 protein levels were quantified on AMs using immunostaining techniques, as described in "Methods". The boxes in the box-plots show the medians and $25^{\text {th }}$ and $75^{\text {th }}$ percentiles, and the whiskers show the $10^{\text {th }}$ and $90^{\text {th }}$ percentiles; $n=5$ mice/group with 200-300 cells analyzed/mouse. Data were analyzed using a Kruskal-Wallis one-way ANOVA followed by pair-wise testing with Mann-Whitney $U$ tests. ${ }^{*} P=0.008$ versus air group. d, e AMs were isolated from naïve WT mice and incubated for $24 \mathrm{~h}$ at $37^{\circ} \mathrm{C}$ with varying concentrations of CS extract (CSE; d) or for varying times with $2.5 \%$ CSE (e). Cells were then fixed and immunostained for surface Adam15, as described in "Methods". Data are mean \pm SEM; $n=150-200$ cells/group. The experiments shown are representative of at least three independent experiments yielding similar results. Data were analyzed using a one-way ANOVA followed by pair-wise testing with two-tailed Student's $t$ tests. ${ }^{*} P<0.001$ compared to unstimulated cells. $\mathbf{f}, \mathbf{g}$ CD $8^{+}$lymphocytes were isolated from the spleens of naïve WT mice and incubated at $37^{\circ} \mathrm{C}$ for $2 \mathrm{~h}$ with varying concentrations of CSE (f) or with or without $2.5 \%$ CSE for varying times up to $60 \mathrm{~min}(\mathbf{g})$. Cells were fixed and immunostained for surface Adam15. Data are mean $\pm \mathrm{SEM} ; n=150-200$ cells/group. The experiments shown are representative of at least three independent experiments yielding similar results. Data were analyzed using a one-way ANOVA followed by pair-wise testing with two-tailed Student's $t$ tests. ${ }^{*} P<0.001$ compared to unstimulated cells. h Steady state ADAM15 mRNA levels were measured in normal human bronchial epithelial cells (HBECs) incubated at $37^{\circ} \mathrm{C}$ for varying times with or without $2.5 \%$ CSE using quantitative real-time PCR. Data are mean \pm SD; $n=3$ experiments. Data were analyzed using a one-way ANOVA followed by pair-wise testing with two-tailed Student's $t$ tests. ${ }^{*} P<0.03$ versus cells incubated without CSE. $\mathbf{i}$, $\mathbf{j}$ human monocytes and CD8 ${ }^{+} \mathrm{T}$ cells were isolated from the blood of healthy donors by positive section for CD14 or CD8 $\alpha$ using immunomagnetic beads. Monocytes and CD8 ${ }^{+} \mathrm{T}$ cells were incubated with or without CSE for varying times and/or with concentrations of CSE. Cells were fixed and non-permeabilized cells were immunostained for surface ADAM15 as described in Methods. Data are mean $\pm \mathrm{SEM} ; n=150-200$ cells/group. The experiments shown are representative of at least three independent experiments yielding similar results. Data were analyzed using a one-way ANOVA followed by pair-wise testing with two-tailed Student's $t$ tests. ${ }^{*} P<0.001$ compared to unstimulated cells.

$\mathrm{CD}^{+} \mathrm{T}$ cells isolated from unchallenged WT mice (Fig. 1f). Maximal increases in surface Adam 15 levels occurred rapidly (within 30 min of adding CSE [(Fig. 1g]) as reported for release of perforin and granzyme $B$ from the lytic granules of antigenactivated $\mathrm{CD}^{+}{ }^{\text {T cells. }}{ }^{15}$ CSE also increased ADAM15 expression in human cells including ADAM15 transcript levels in human bronchial epithelial cells (HBECs), and surface ADAM protein levels on monocytes and $\mathrm{CD}^{+}{ }^{+} \mathrm{T}$ cells in vitro (Fig. $1 \mathrm{~h}-\mathrm{j}$ ).

Adam $15^{-1-}$ mice develop exaggerated CS-induced COPD-like disease and pulmonary inflammation

Adam $15^{-1-}$ mice have normal lung development and a normal phenotype in the unchallenged state. ${ }^{18}$ When exposed to CS for 6 months, Adam $15^{-1-}$ mice had greater emphysema development (Fig. 2a, b) and small airway fibrosis (Fig. 2c, d), and more a-SMApositive cells in their small airways (Fig. 2e, f) than WT mice. WT and Adam $15^{-1-}$ mice had similar CS-induced airway mucus metaplasia (Fig. E2).

Inhaling CS induces a chronic pulmonary inflammatory response in mice and humans which promotes airspace enlargement. ${ }^{2,15,19}$ Thus, we evaluated whether the exaggerated airspace enlargement observed in CS-exposed Adam $15^{-1-}$ mice is associated with increased pulmonary inflammation. CS-exposed Adam $15^{-1-}$ mice had greater increases in bronchoalveolar lavage (BAL) total leukocyte, macrophage, and lymphocyte counts than CS-exposed WT mice (Fig. 3a-c). Adam $15^{-1-}$ mice had lower increases in BAL PMN counts than WT mice after 6 months CS exposure (Fig. E3A). Mean total blood leukocyte and leukocyte subset counts were similar in air- and CS-exposed Adam $15^{-1-}$ and WT mice (Table E1).

When exposed to CS for 1 and/or 3 months, Adam $15^{-1-}$ mice had a higher percentage of $\mathrm{CD}^{+}$lymphocytes (Fig. 3d), $\mathrm{CD}^{+}$ lymphocytes (Fig. 3e), and CD3-positive NKT cells (Fig. 3f) but a lower percentage of lung $\mathrm{CD}^{+} \mathrm{CD} 4^{+} \mathrm{Foxp}^{+} \mathrm{T}$ regulatory cells (Tregs; Fig. 3g) in enzymatic lung digest samples than WT mice. The percentage of $B$ cells in lung digest samples was lower in Adam $15^{-1-}$ mice than WT mice after 1 month of CS exposure, but similar after 3 months of CS exposure (Fig. 3h). Adam $15^{-/-}$mice had a lower percentage of CD3-negative natural killer (NK) cells in enzymatic lung digest samples when exposed to air or CS for up to 3 months than WT mice (Fig. E3B). Flow cytometry dot plots are shown in Fig. E4.
Lung Ccl-2, Cxcl-10, Gm-csf, and Mmp-12 levels were higher in CS-exposed Adam $15^{-1-}$ versus WT mice (Fig. 4a-d). Lung II-10, II-6, and II- $1 \beta$ levels were lower in CS-exposed Adam $15^{-1-}$ versus WT mice (Fig. 4e-g). Lung Tgf- $\beta$, Tnf- $\alpha, C \mathrm{Cl}-5, \mathrm{M}-\mathrm{csf}$, and $\mathrm{Ccl}-3$ levels were similar in CS-exposed WT and Adam $15^{-1-}$ mice (Fig. E5). CS exposure increased lung levels of granzyme $\mathrm{A}\left(\mathrm{a} \mathrm{CD} 8^{+} \mathrm{T}\right.$ cell product which injures pulmonary epithelial cells ${ }^{16}$ ) in Adam $15^{-1-}$ but not WT mice (Fig. 4h).

Adam 15 deficiency in leukocytes leads to exaggerated pulmonary inflammation and COPD-like disease

Adam 15 is expressed by leukocytes and lung parenchymal cells. Thus, Adam 15 BM chimeras were generated and exposed to air or CS for 6 months to identify the Adam15-deficient cells that drive the greater CS-induced COPD-like disease. Adam 15 deficiency in leukocytes (not lung parenchymal cells) increased CS-induced emphysema development (Fig. 5a), small airway fibrosis (Fig. 5b) and lung inflammation as follows: (1) WT recipients transplanted with Adam $15^{-1-}$ BM had greater CS-induced emphysema, small airway fibrosis, and lung macrophage counts (Fig. 5c) than WT recipients transplanted with WT BM; and (2) Adam $15^{-1-}$ recipients transplanted with WT BM had reduced CS-induced emphysema development, small airway fibrosis, and macrophage counts than Adam $15^{-1-}$ recipients transplanted with Adam $15^{-1-}$ BM (Fig. 5a-c).

As $\mathrm{Cd}^{-1-}$ mice are protected from CS-induced accumulation of macrophages in the lung and emphysema development, ${ }^{15}$ Adam $15^{-1-} x \mathrm{Cd} 8^{-1-}$ compound mutant mice were generated and exposed to air or CS for up to 6 months. Adam 15 deficiency in CD8 ${ }^{+}$ $T$ cells was crucial for driving the enhanced pulmonary inflammation and emphysema development in Adam $15^{-/-}$mice as CS-exposed Adam $15^{-1-} x C d 8^{-1-}$ mice had lower BAL total leukocyte, macrophage, and lymphocyte counts and reduced emphysema development compared with CS-exposed Adam $15^{-/-}$or WT mice (Fig. 6).

Adam15 is not required for $\mathrm{CD8}^{+} \mathrm{T}$ cell or macrophage adhesion or migration

ADAM15 increases glomerular mesangial cell migration and cell-cell interactions of a fibroblast cell line. ${ }^{12,14}$ Whether Adam15 regulates the adhesion of migration of leukocytes is not known. WT and Adam $15^{-1-}$ macrophages and $\mathrm{CD}^{+} \mathrm{T}$ cells did not differ 

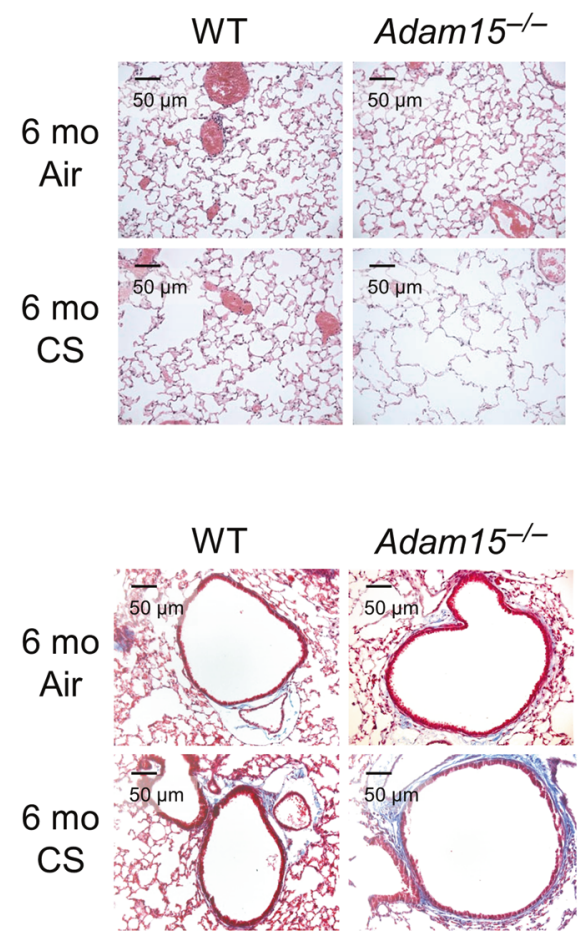

e

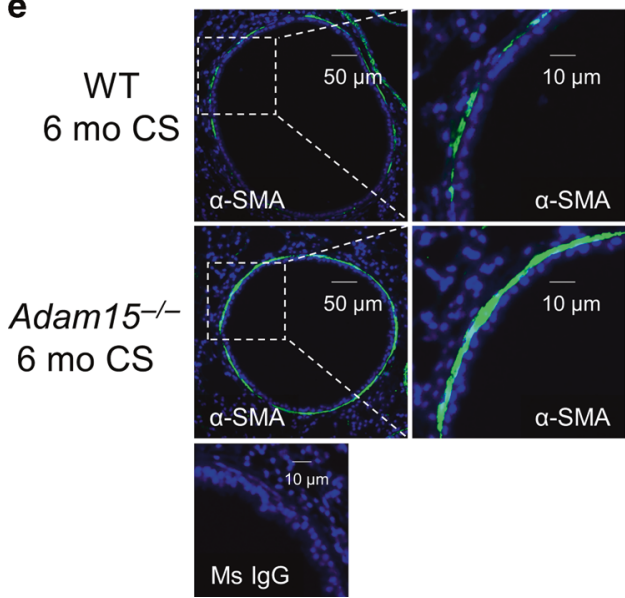

b

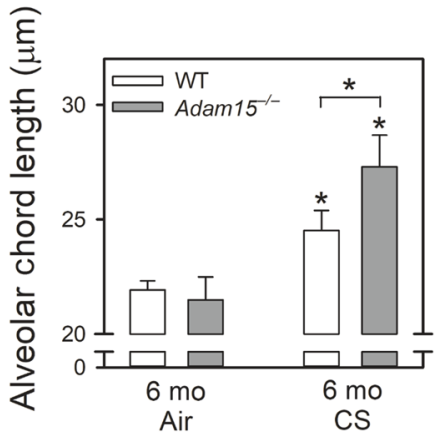

d
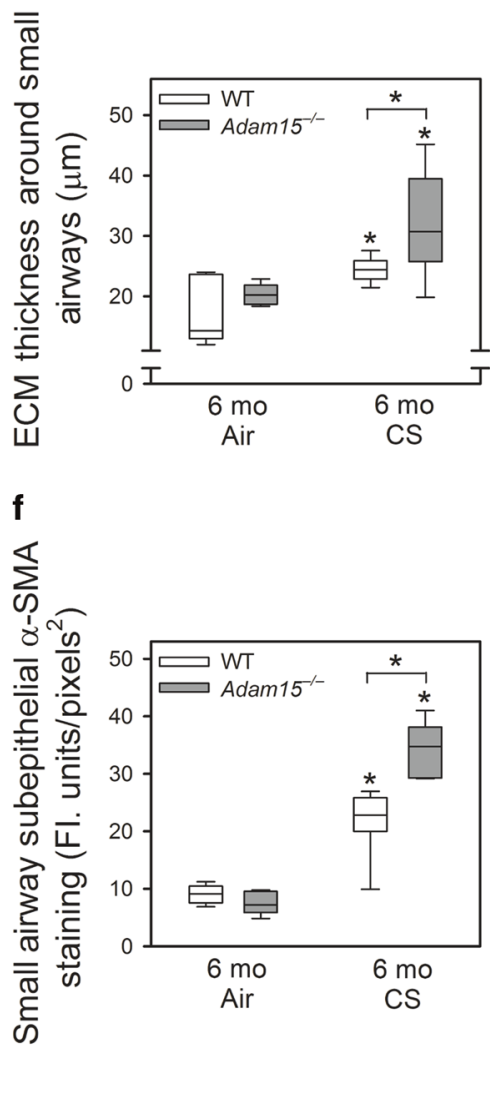

Fig. 2 Adam 15 deficiency in mice increases CS-induced emphysema development and small airway fibrosis. Adult Adam15 ${ }^{-1-}$ mice and WT mice were exposed to air or CS 6 days-a-week for 6 months (Mo). a Images of hematoxylin and eosin-stained inflated lung sections from mice. b Airspace size was quantified in Gills-stained lung sections using Scion image analysis software, as described in the "Methods". The mean chord length is the average of the horizontal and vertical distances between the alveolar walls. Data are mean \pm SD; $n=8-11$ mice exposed to air and 11-14 mice exposed to CS. Data were analyzed using a one-way ANOVA followed by pair-wise testing with two-tailed Student's $t$ tests. ${ }^{*} P \leq 0.035$ versus the same genotype exposed to air or the group indicated. c Representative images of extracellular matrix (ECM) deposition around small airways (300-699 $\mu \mathrm{m}$ in diameter) in Masson Trichrome-stained lung sections from WT and Adam15 ${ }^{-1-}$ mice exposed to air or CS for 6 months. d The thickness of the ECM protein layer deposited around small airways was quantified using morphometry methods, as described in the "Methods". The boxes in the box-plots show the medians and $25^{\text {th }}$ and $75^{\text {th }}$ percentiles, and the whiskers show the $10^{\text {th }}$ and $90^{\text {th }}$ percentiles; $n=7-10$ mice/group. Data were analyzed using a Kruskal-Wallis one-way ANOVA followed by pair-wise testing with Mann-Whitney $U$ tests. ${ }^{*} P \leq 0.038$ versus the same genotype exposed to air or the group indicated. e Murine lung sections were immunostained for $\alpha$-smooth muscle actin ( $\alpha$-SMA, a marker of myofibroblasts or smooth muscle cells) around small airways of WT and Adam $15^{-1-}$ mice that were exposed to air or CS for 6 months. Nuclei were counterstained blue with 4',6-diamidino-2-phenylindole. As a control, lung sections were stained with a non-immune murine (Ms) lgG (bottom panel). The merged images shown in e are representative of 5-7 mice/group. $f$ Box-plots of $\alpha$-SMA staining that was quantified per unit area of the walls of small airways having a luminal diameter of 300-699 $\mu \mathrm{m}$ using MetaMorph software (5 air-exposed mice/group and 6-7 CS-exposed mice/group). The boxes in the box-plots show the medians and $25^{\text {th }}$ and $75^{\text {th }}$ percentiles, and the whiskers show the $10^{\text {th }}$ and $90^{\text {th }}$ percentiles. Data were analyzed using a Kruskal-Wallis oneway ANOVA followed by pair-wise testing with Mann-Whitney $U$ tests. ${ }^{*} P \leq 0.005$ versus air-exposed mice belonging to the same genotype or the group indicated. 
a

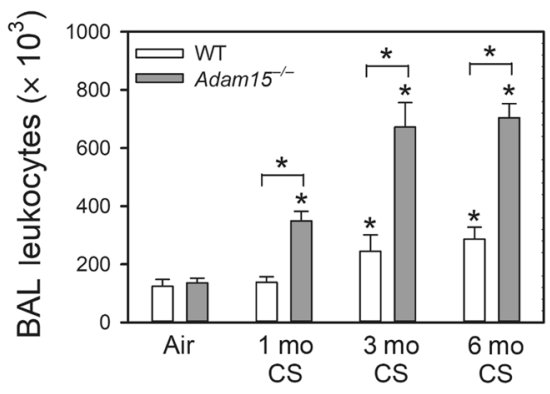

C

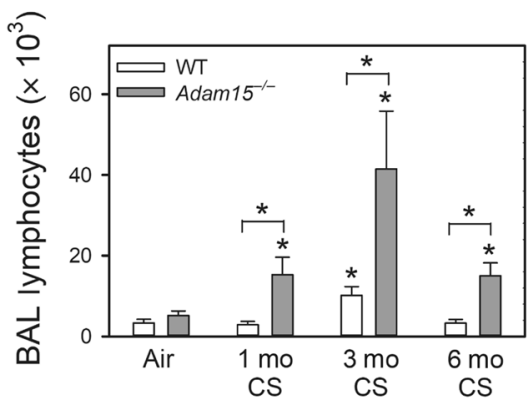

e

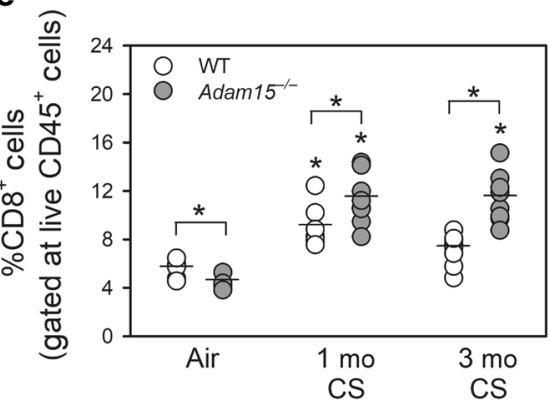

g

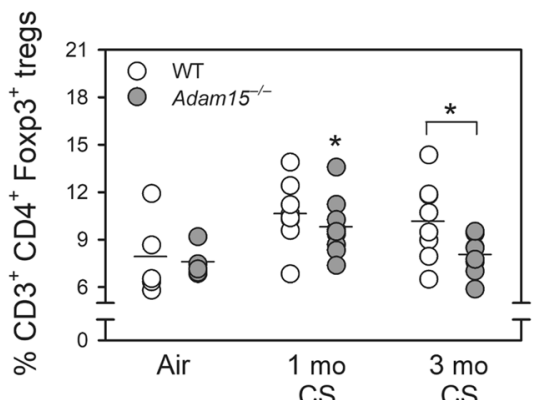

b

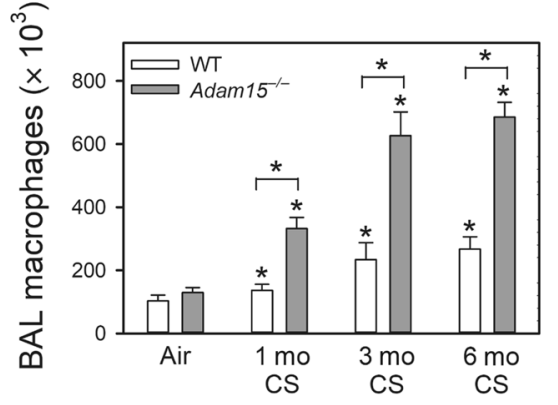

d

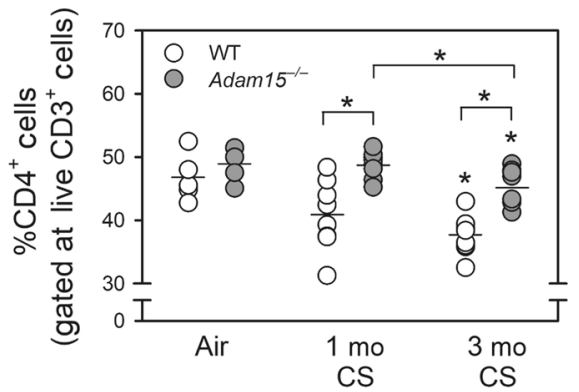

f

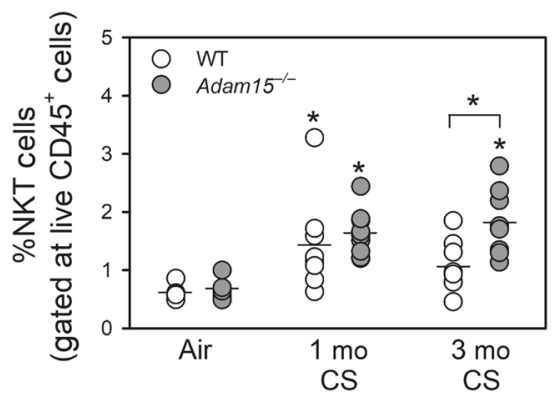

h

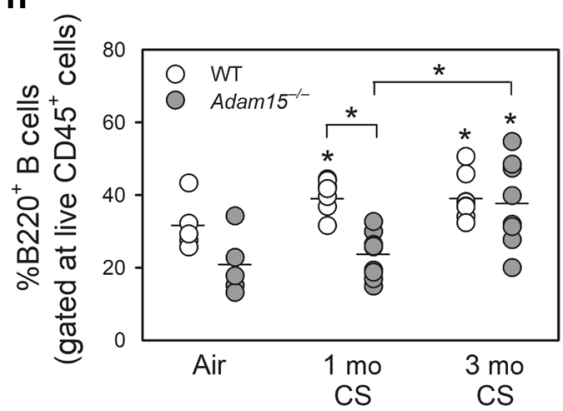

Fig. 3 Deficiency of Adam 15 in mice increases CS-induced lung inflammation. Wild-type (WT) and Adam $15^{-1-}$ mice were exposed to air or CS for 1 month (mo), 3 months or 6 months. Absolute numbers of all leukocytes (a), macrophages (b), and lymphocytes (c) were counted in bronchoalveolar lavage (BAL) samples. Data are mean $\pm \mathrm{SEM} ; n=12-14$ mice exposed to air and 6-11 mice exposed to CS. Data were analyzed using a one-way ANOVA followed by pair-wise testing with two-tailed Student's $t$ tests. ${ }^{*} P \leq 0.005$ versus the same genotype exposed to air or the group indicated. d, h WT and Adam $15^{-1-}$ mice were exposed to air or CS for 1 or 3 months, lungs were enzymatically digested, and leukocytes were immunostained for markers of lymphocyte subsets including CD4 ${ }^{+}$lymphocytes (d), CD8 ${ }^{+}$lymphocytes (e), CD3-positive NKT cells (f), Tregs (g), and B cells (h). Lymphocyte subsets were quantified using flow cytometry. Data are mean \pm SD; $n=5$ mice exposed to air and 8-9 mice exposed to CS. Data were analyzed using a one-way ANOVA followed by pair-wise testing with two-tailed Student's $t$ tests. ${ }^{*} P<0.05$ air-exposed mice belonging to the same genotype or the group indicated.

in their capacity to adhere to an ECM protein (Fig. E6) or migrate toward chemoattractants (Fig. E7) in vitro. Thus, it is unlikely that Adam 15 deficiency increases CS-induced pulmonary inflammation by increasing the trans-endothelial migration of monocytes or $\mathrm{CD}^{+} \mathrm{T}$ cells into the lung.
Adam15 deficiency in $\mathrm{CD}^{+}{ }^{+} \mathrm{T}$ cells and macrophages abrogates CSinduced activation of the mitochondrial apoptosis pathway to increase cell survival by reducing CS-induced inactivation of mTOR signaling CS-induced apoptosis of leukocytes regulates leukocyte accumulation in the lung. ${ }^{2}$ Compared with CS-exposed WT mice, 
a

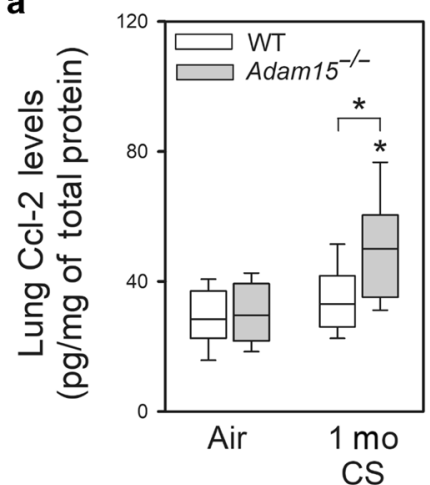

d

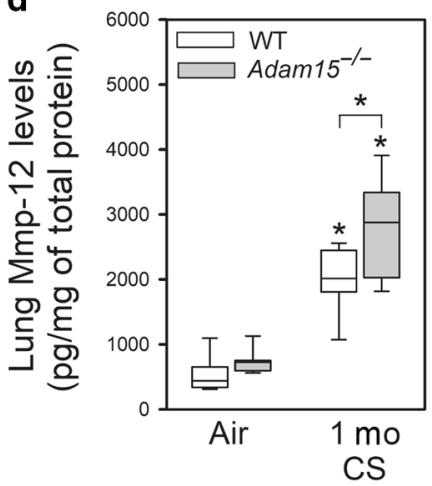

b

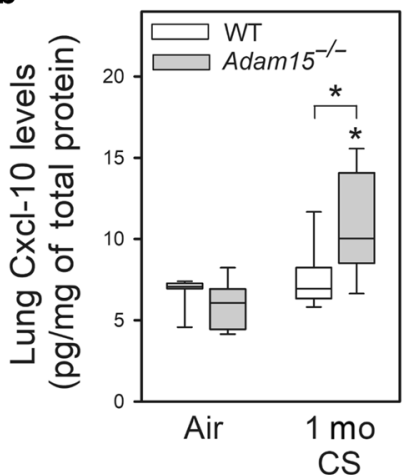

e

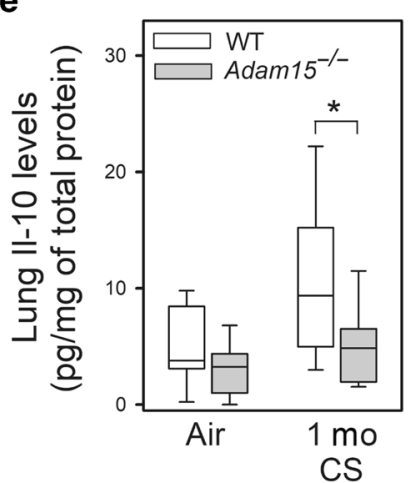

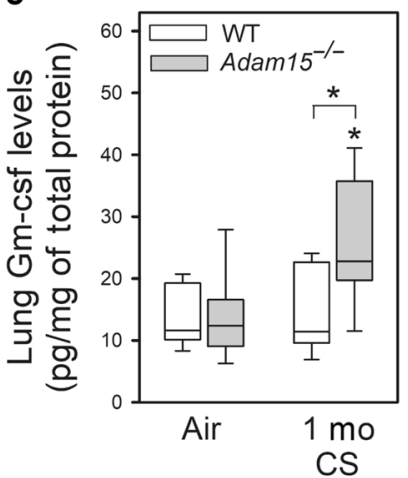

f

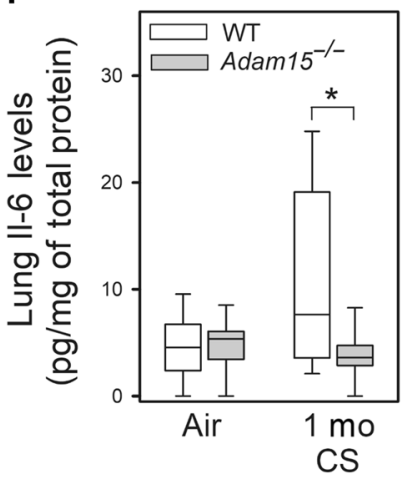

g

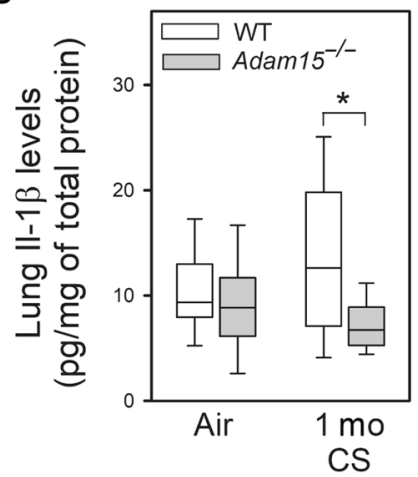

h

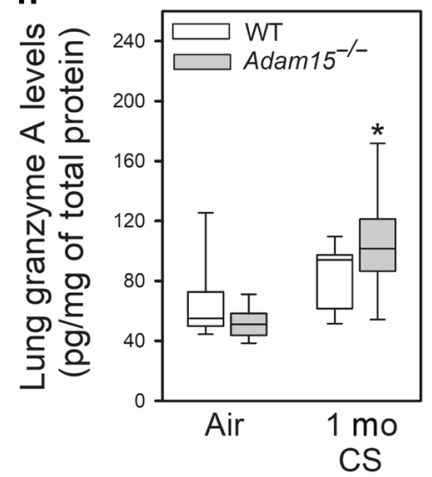

Fig. 4 Deficiency of Adam15 in mice increases lung levels of mediators that promote CS-induced lung inflammation and destruction. WT and Adam $15^{-1-}$ mice were exposed to air or CS for 1 month (mo), lung levels of Ccl-2 (a; $n=10-12$ mice/group), Cxcl-10 (b; $n=7$ mice/group), Gm-csf (c; $n=10-12$ mice/group), Mmp-12 (d; $n=8$ mice/group), II-10 (e; $n=10-12$ mice/group), II-6 (f; $n=14-22$ mice/group), II-1 $\beta$ (g; $n=$ 13-22 mice/group) and granzyme A (h; $n=8$ mice/group) were measured in homogenates of lung samples using commercial ELISA kits. Data were normalized to total protein levels measured in the same samples. The boxes in the box-plots show the medians and $25^{\text {th }}$ and $75^{\text {th }}$ percentiles, and the whiskers show the $10^{\text {th }}$ and $90^{\text {th }}$ percentiles. Data were analyzed using a Kruskal-Wallis one-way ANOVA followed by pairwise testing with Mann-Whitney $U$ tests. ${ }^{*} P \leq 0.025$ versus air-exposed mice belonging to the same genotype or the group indicated.

CS-exposed Adam $15^{-/-}$mice had reduced rates of apoptosis in their alveolar macrophages (AMs) and $\mathrm{CD} 8^{+} \mathrm{T}$ cells (assessed as lower intracellular active caspase-3 levels; Fig. 7a, b, respectively) and reduced CS-induced activation of the mitochondrial apoptosis pathway in their AMs (as assessed by a lower percentage of cells that did not stain with the mitochondrial dye, JC-1 indicative of loss of mitochondrial membrane potential; Fig. 7c). CSE-treated macrophages and $\mathrm{CD}^{+} \mathrm{T}$ cells isolated from naive Adam $15^{-/-}$ mice exhibited reduced activation of the mitochondrial apoptosis pathway in vitro (Fig. 7d, e).

The PI3 kinase-Akt-mTOR pathway inhibits activation of the mitochondrial apoptosis pathway by increasing the expression of anti-apoptotic Bcl-2 family members, especially intracellular myeloid cell leukemia sequence-1 (Mcl-1) in macrophages and $\mathrm{T}$ cells. ${ }^{20,21}$ Translocation of $\mathrm{Bcl}-2$ proteins to the mitochondrial membrane prevents mitochondrial membrane depolarization and cytochrome $C$ release. ${ }^{22}$ The mTOR-Mcl- 1 pathway prosurvival pathway was measured in $\mathrm{CD}^{+} \mathrm{T}$ cells isolated from the lungs mice that had been exposed to CS for 3 months, or in macrophages isolated from unchallenged mice and exposed to CSE in vitro. $\mathrm{CD}^{+} \mathrm{T}$ cells isolated from the lungs of Adam $15^{-/-}$ mice had greater intracellular levels of phosphorylated ribosomal protein S6 (P-S6; a marker of mTOR signaling; Fig. 8a, b) and higher intracellular levels of Mcl-1 (Fig. 8c, d). CSE exposure substantially reduced intracellular levels of P-S6 (Fig. 8e) and Mcl-1 in WT but not Adam 15 $5^{-1-}$ macrophages in vitro (Fig. 8f, g). 
a

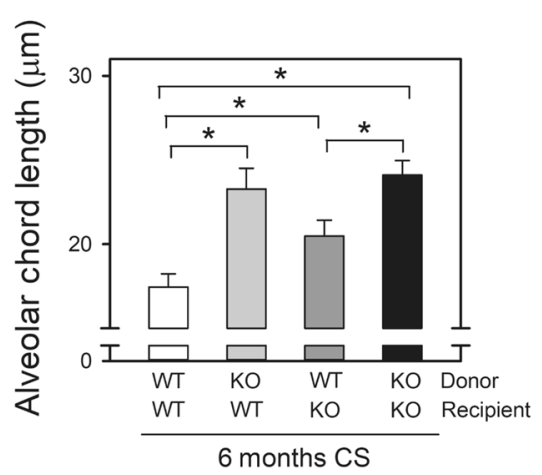

C

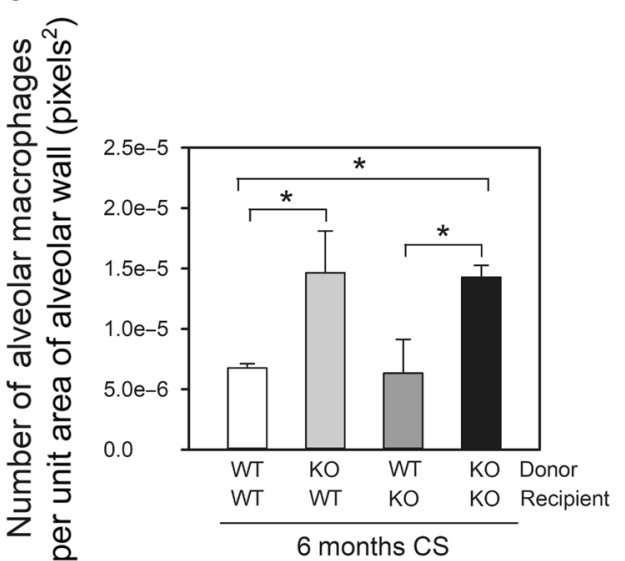

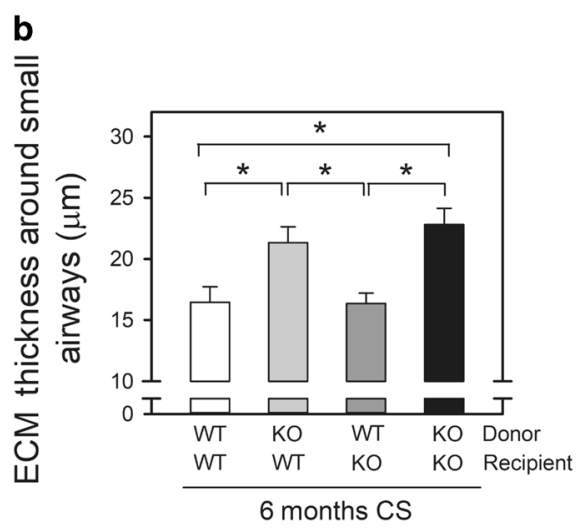

Fig. 5 Deficiency of Adam 15 in bone marrow (BM)-derived leukocytes drives the exaggerated COPD-like disease detected in CS-exposed Adam $15^{-I-}$ mice. a-c Four groups of Adam15 BM chimeric mice (WT BM transplanted into WT recipients [WT WT], Adam15 ${ }^{-1-}$ BM transplanted into WT recipients [KO WT], WT BM transplanted into Adam $15^{-1-}$ recipients [WT KO], and Adam $15^{-1-}$ BM transplanted into Adam $15^{-/-}$recipients [KO KO]) were exposed to air or CS for 6 months. Panel a shows the mean alveolar chord lengths measured in the CSexposed mice. The mean chord length in air-exposed mice was 14-19 $\mu \mathrm{m}$ in all groups (data not shown). Data are mean \pm SEM; $n=4-6$ mice/ group. Data were analyzed using a one-way ANOVA followed by pair-wise testing with two-tailed Student's $t$ tests. ${ }^{*} P<0.05$ versus the group indicated. $\mathbf{b}$ the thickness of the ECM protein layer deposited around small airways was quantified in microns, as described in Methods. Data are mean \pm SEM; $n=5-8$ mice/group. Data were analyzed using a one-way ANOVA followed by pair-wise testing with two-tailed Student's $t$ tests. ${ }^{*} P \leq 0.022$ versus the group indicated. $\mathbf{c}$ The number of alveolar macrophages in lung sections normalized to alveolar wall area was measured, as described in the "Methods". Data are mean \pm SEM; $n=5-6$ mice/group. Data were analyzed using a one-way ANOVA followed by pair-wise testing with two-tailed Student's $t$ tests. ${ }^{*} P<0.05$ versus the group indicated.

Thus, Adam15 deficiency promotes the survival of $\mathrm{CD}^{+} \mathrm{T}^{\mathrm{T}}$ cells and macrophages in the CS-exposed lungs by rendering these cells resistant to CS-triggered activation of the mitochondrial apoptosis pathway by preserving their mTOR-Mcl-1 signaling when they are exposed to CS.

ADAM15 overexpression reduces CSE-induced activation of the mitochondrial apoptosis pathway in monocyte/macrophage-like cells

ADAM15 was overexpressed in human differentiated THP-1 (monocyte-macrophage-like) cells using a vector containing an ADAM15 complementary DNA (cDNA) and electroporation. Robust ADAM15 expression was detected using real-time quantitative polymerase chain reaction (PCR) in THP-1 cells $24 \mathrm{~h}$ after electroporation but not in cells treated with the control vector (Fig. 9a). THP-1 cells overexpressing ADAM15 had increased CSEinduced activation of the mitochondrial apoptosis pathway (as assessed by a higher percentage of cells that did not stain with JC-1; Fig. 9b) and reduced intracellular MCL-1 protein levels compared with cells treated with the control vector (Fig. 9c, d). Thus, ADAM15 overexpression promotes CSE-induced inactivation of mTOR-Mcl-1 signaling to increase the mitochondrial apoptosis pathway in monocyte/macrophage-like cells.

\section{DISCUSSION}

This study links Adam 15 to the pathogenesis of COPD-like disease, leukocyte function, and the mTOR pathway in CS-exposed mice for the first time. Acute CS exposure increased lung Adam15 transcript and protein levels in WT mice, and Adam15 was localized to pulmonary macrophages, $\mathrm{CD} 8^{+} \mathrm{T}$ cells, epithelial cells, and airway a-ASM-positive cells in CS-exposed WT mice. When exposed to CS for 6 months, Adam $15^{-/-}$mice developed exaggerated pulmonary inflammation, emphysema development, and small airway fibrosis compared with WT mice. Adam15 deficiency in BM-derived leukocytes (especially $\mathrm{CD} 8^{+} \mathrm{T}$ cells) was crucial for driving the exaggerated COPD-like disease in CSexposed Adam $15^{-1-}$ mice. Adam 15 deficiency increased $\mathrm{CD}^{+}$ $\mathrm{T}$ cell and macrophage counts in the CS-exposed murine lung by increasing the resistance of these cells to CS-induced activation of the mitochondrial apoptosis pathway thereby increasing the survival of these cells in the lung. Adam 15 deficiency reduced activation of the mitochondrial apoptosis pathway in pulmonary $\mathrm{CD}^{+} \mathrm{T}$ cells and macrophages by abrogating CS-induced inactivation of mTOR signaling in $\mathrm{CD}^{+} \mathrm{T}$ cells and macrophages. The greater mTOR signaling in CS-exposed $\mathrm{CD}^{+} \mathrm{T}$ cells and macrophages leads to higher intracellular levels of Mcl-1 (a Bcl-2 prosurvival protein) which translocates to the mitochondrial 
a

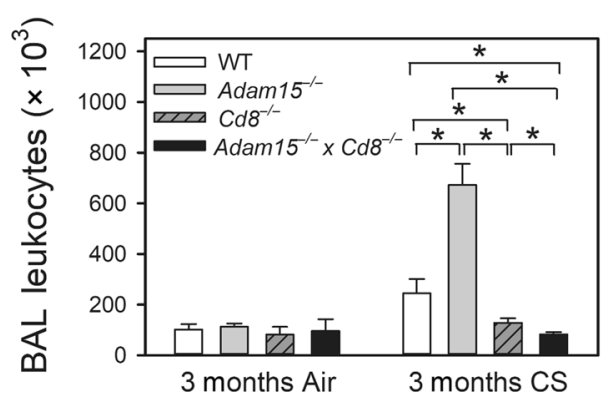

C

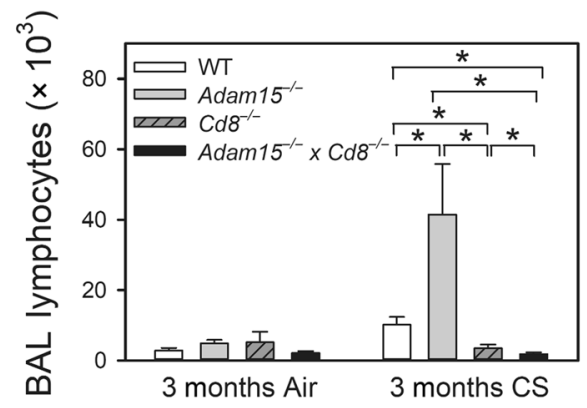

b

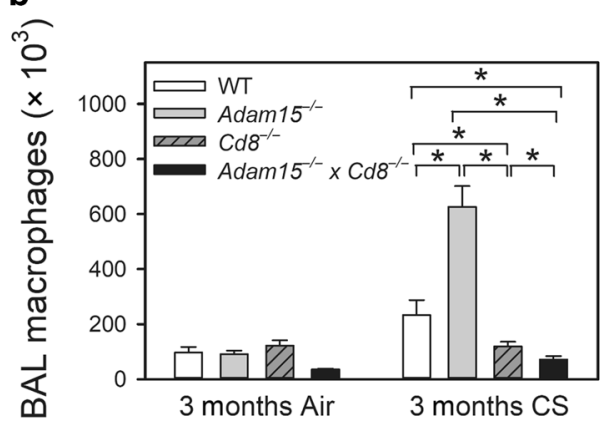

d

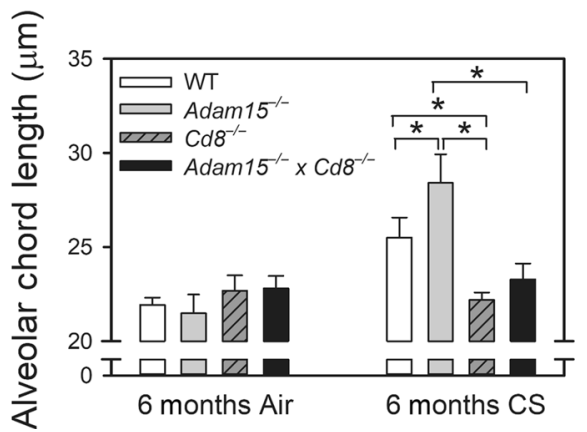

Fig. 6 Deficiency of Adam 15 in $\mathrm{CD8}^{+} \mathrm{T}$ cells is crucial for driving the exaggerated lung inflammation and destruction detected in CSexposed Adam $15^{-1-}$ mice. Adult WT mice, Adam $15^{-1-}$ mice, $C d 8^{-1-}$ mice, and Adam $15^{-1-} \times C d 8^{-1-}$ compound mutant mice were exposed to air or CS 6 days-a-week for 3 months or 6 months. Absolute numbers of all leukocytes (a), macrophages (b), and lymphocytes (c) were counted in bronchoalveolar lavage (BAL) samples. Data are mean \pm SEM; $n=7-17$ mice exposed to air for 3 months air and 6-11 mice exposed to CS for 3 months. Data were analyzed using a one-way ANOVA followed by pair-wise testing with two-tailed Student's $t$ tests. ${ }^{*} P<0.05$ versus the group indicated. D: alveolar chord length was measured on lung sections as a measure of airspace enlargement. Data are mean \pm SEM; $n=$ 5-11 mice exposed to air for 6 months and 10-16 mice exposed to CS for 6 months. Data were analyzed using a one-way ANOVA followed by pair-wise testing with two-tailed Student's $t$ tests. ${ }^{*} P<0.05$ versus the group indicated.

membrane to reduce loss of mitochondrial membrane potential, downstream caspase-3 activation, and apoptosis (Fig. 10). Conversely, ADAM15 overexpression in THP-1 cells increases CSEinduced inactivation of $\mathrm{mTOR}-\mathrm{MCL}-1$ signaling and promotes CSE-induced activation of the mitochondrial apoptosis pathway.

ADAM15 has not been studied previously in COPD. ADAM15 gene expression is upregulated macrophages and fibroblasts when they are activated. ${ }^{9}$ Although ADAM 15 was reported not to be expressed by $\mathrm{CD}^{+} \mathrm{T}$ cells in healthy subjects, ${ }^{23}$ we report increased Adam15 staining in $\mathrm{CD}^{+} \mathrm{T}$ cells in CS-exposed WT lungs and on the surface of CSE-activated murine $\mathrm{CD} 8^{+} \mathrm{T}$ cells in vitro.

The increased Adam15 gene expression in the lungs of WT mice that were exposed acutely to CS could be mediated by the increased lung levels of pro-inflammatory mediators present in their lungs. (Epi)genetic processes could also increase Adam 15 expression in CS-exposed murine lungs. The murine Adam15 promoter contains three Sp1-binding sites. ${ }^{24}$ CS increases Sp1 binding to the promoter regions of other genes to activate their transcription. ${ }^{25}$ Whether Sp1 binding to the Adam 15 promoter contributes to the increased Adam 15 expression observed in CSexposed WT murine lungs mice is unclear. Single-nucleotide polymorphisms in the ADAM15 locus are linked to rheumatoid arthritis, ${ }^{26}$ but to our knowledge have not linked to human COPD. MicroRNAs (miRNAs) regulate gene transcription in human and murine COPD. ${ }^{27}$ MiR-147b binds the $3^{\prime}$ untranslated region of the ADAM15 mRNA in human endothelial cells to reduce ADAM15 expression in these cells. ${ }^{28}$ Whether other miRNAs increase Adam 15 expression in CS-exposed murine lungs will be investigated in our future studies.
CSE increased ADAM15 surface levels on human monocytes and $\mathrm{CD}^{+} \mathrm{T}$ cells and ADAM15 transcript levels in HBECs in vitro indicating that the expression data obtained from murine cells are translatable to humans. In publicly-available Gene Expression Omnibus (GEO) microarray and RNA sequencing data-sets, ADAM15 transcript levels were higher in bronchial epithelial cells from COPD patients versus non-smokers and smokers but were not related to GOLD stage (GSE5058 and GSE 63127; data not shown). ADAM15 transcript levels in AMs were higher in smokers versus non-smokers, but not increased in AMs from COPD patients (GSE130928; data not shown).

CS-exposed Adam $15^{-1-}$ mice developed exaggerated COPDlike disease suggesting that Adam15 has protective activities in lungs exposed chronically to CS. However, WT mice exposed to CS for up to 2 months had increased lung levels of Adam15. Heme oxygenase-1 (HO-1) is a cyto-protective protein which is upregulated during various acute insults in humans and mice ${ }^{29,30}$ including hypoxia- and ischemia-reperfusion (IR)-induced tissue injury. However, $\mathrm{HO}_{-1}^{-1-}$ mice have exaggerated disease compared with WT mice in these injury models. ${ }^{29,30}$ While 2 months of CS exposure increased lung Adam15 protein levels in WT mice 7fold, 6 months of CS exposure did not further increase Adam15 lung levels. Thus, increases in Adam15 lung levels induced by acute CS exposures may represent an acute cyto-protective stress response similar to the increases in $\mathrm{HO}-1$ tissue levels observed in WT mice with acute hyperoxia- or IR-induced tissue injury. ${ }^{31,32}$ We hypothesize that the failure to upregulate Adam15 lung levels further during more chronic CS exposures contributes to inadequate protection of the lungs of WT mice from the deleterious effects of CS. The absence of Adam15 in the lungs 


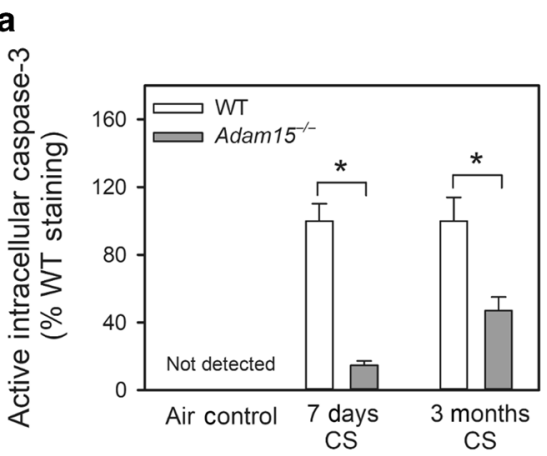

b
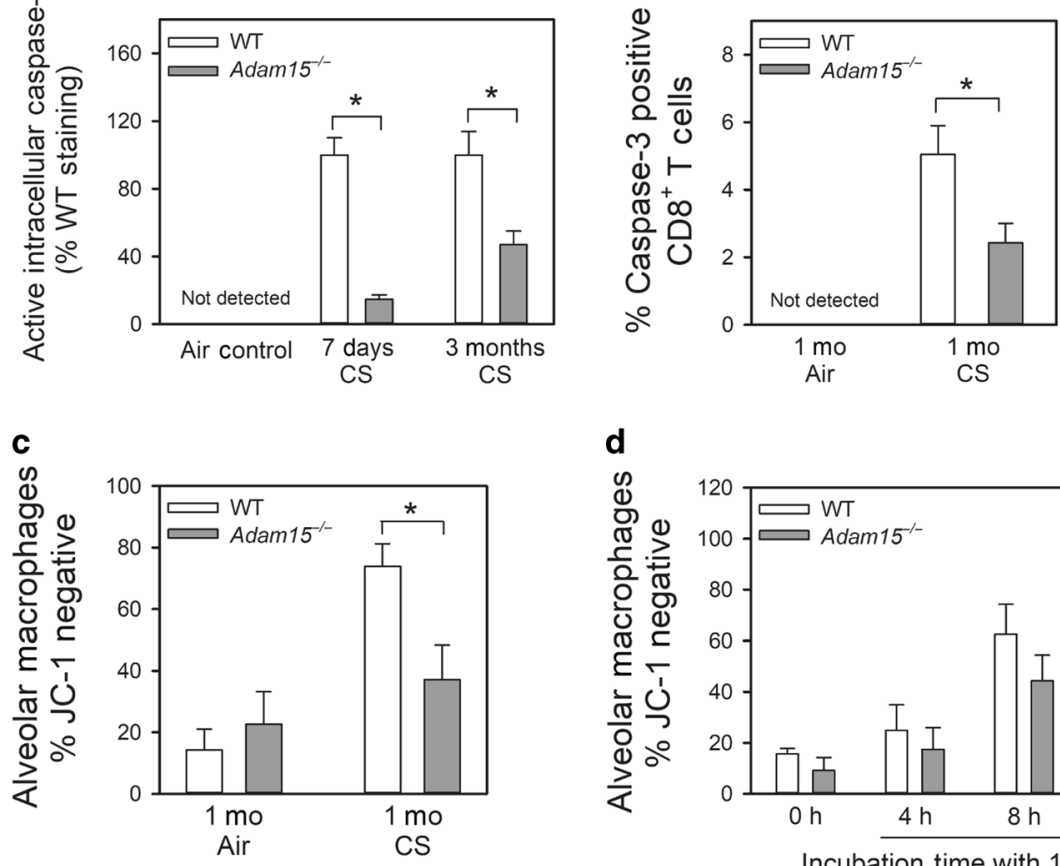

d
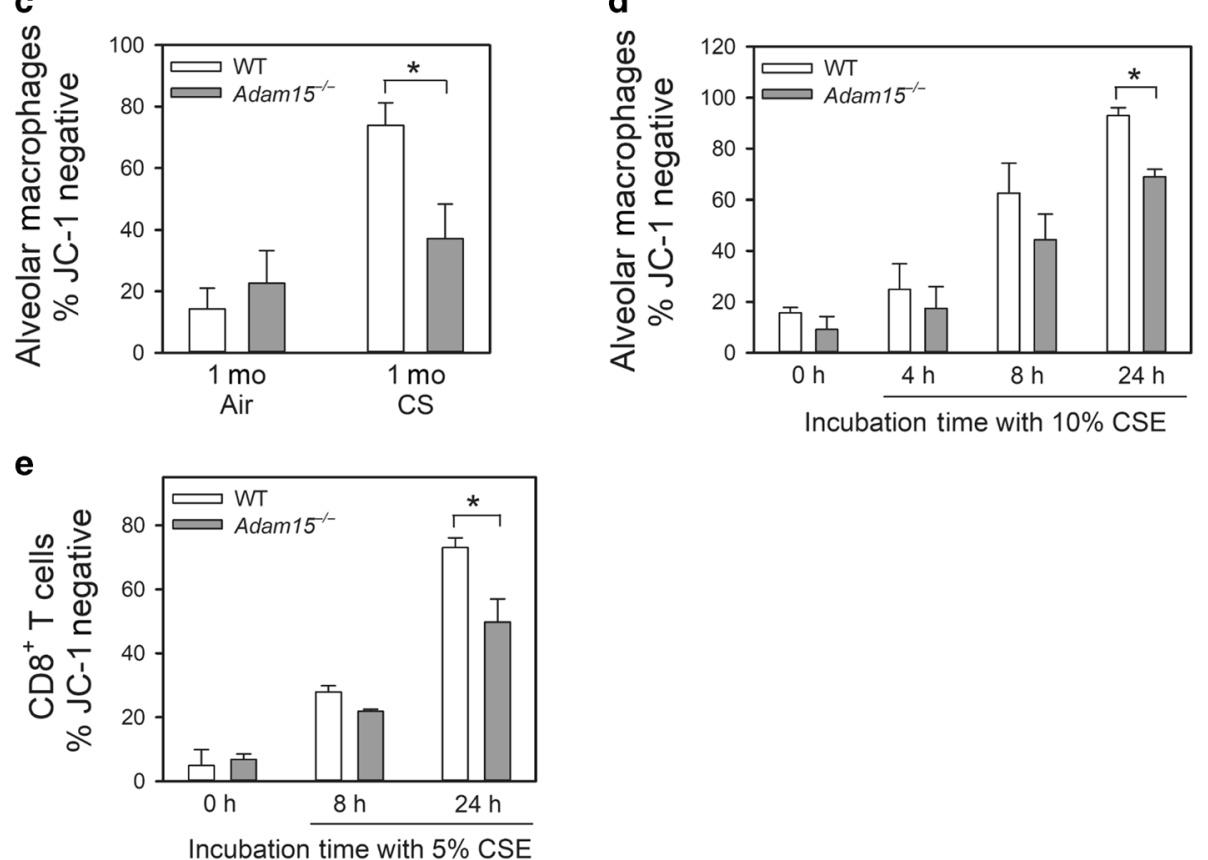

Fig. 7 Deficiency of Adam15 in $\mathrm{CD8}^{+} \mathrm{T}$ cells and macrophages protects the cells from CS-induced activation of the mitochondrial apoptosis pathway in vivo and in vitro. a Alveolar macrophages (AMs) were isolated from the lungs of WT and Adam15 $5^{-/-}-1-$ mice that had been exposed to air or CS for 1 week or 3 months (Mo) using BAL. $\mathbf{b}$ CD8 ${ }^{+}$T cells were isolated from the lungs of WT and Adam $15^{-1-}$ mice exposed to air or CS for 1 month as described in "Methods". a, b Immediately after the AMs and CD8 ${ }^{+}$T cells were isolated they were fixed and then immunostained for intracellular active caspase-3. Staining was quantified, as described in the "Methods". Active caspase-3 was not detected in macrophages or CD8 ${ }^{+}$T cells isolated from air-exposed WT or Adam $15^{-/-}$mice. Data are mean \pm SEM; $n=5$ mice/group in (a), and $n=4-5$ mice/group in (b). Data were analyzed using a one-way ANOVA followed by pair-wise testing with two-tailed Student's $t$ tests. ${ }^{*} P<$ 0.05 versus the group indicated. c AMs were isolated from the lungs of WT and Adam $15^{-1-}$ mice exposed to air or CS for 1 month using BAL. Activation of the mitochondrial apoptosis pathway was measured as loss of mitochondrial membrane potential (lack of staining of the cells with the mitochondrial dye, JC- 1 which stains only viable mitochondria). Data are mean $\pm \mathrm{SEM} ; n=5$ mice/group. Data were analyzed using a one-way ANOVA followed by pair-wise testing with two-tailed Student's $t$ tests. ${ }^{*} P<0.05$ versus the group indicated. $\mathbf{d}$ AMs isolated from naïve WT and Adam $15^{-I-}$ mice were incubated at $37^{\circ} \mathrm{C}$ for up to $24 \mathrm{~h}$ with or without $10 \% \mathrm{CS}$ extract (CSE). e CD8 ${ }^{+} \mathrm{T}$ cells isolated from the spleens of naïve WT Adam $15^{-1-}$ mice were incubated at $37^{\circ} \mathrm{C}$ for up to $24 \mathrm{~h}$ with or without $5 \% \mathrm{CSE}$. d, e Activation of the mitochondrial apoptosis pathway was measured as loss of mitochondrial membrane potential (lack of staining of the cells with JC-1). Data are mean \pm SEM; $n=$ 3 separate experiments in (d, e). Data were analyzed using a one-way ANOVA followed by pair-wise testing with two-tailed Student's $t$ tests. ${ }^{*} P<0.05$ versus the group indicated.

of Adam $15^{-/-}$mice likely sensitizes them to the development of CS-induced COPD-like disease.

Studies of Adam 15 BM chimeras and Adam $15^{-1-} x C d 8^{-1-}$ mice revealed that Adam 15 deficiency in leukocytes (especially $C D 8^{+}$ T cells) was responsible for the exaggerated pulmonary inflammation and COPD-like disease in CS-exposed Adam $15^{-1-}$ mice. Macrophages are required for CS-induced emphysema development in mice ${ }^{19,33}$ as their products (e.g., Mmp-12 and oxidants) injure the alveolar walls. ${ }^{34}$ However, $\mathrm{CD} 8^{+} \mathrm{T}$ cells are required for macrophage accumulation and activation in the CS-exposed lung ${ }^{15}$ (Fig. 10). $\mathrm{CD}^{+} \mathrm{T}$ cells release ligands (e.g., Cxcl-10) for Cxcr3 expressed on macrophages which induce macrophage activation and Mmp-12 release. $^{15,16} \mathrm{CD}^{+} \mathrm{T}$ cells also induce alveolar epithelial cell apoptosis by releasing perforin and granzymes. ${ }^{15}$ It is noteworthy that CS-exposed Adam $15^{-1-}$ mice had elevated lung levels of $\mathrm{CxCl}-10$, granzyme $\mathrm{A}$, and Mmp-12. CSexposed Adam $15^{-1-}$ mice also had reduced Treg counts in their lungs which may have contributed to their increased pulmonary inflammatory response to CS as Treg release anti-inflammatory molecules such as II-10, ${ }^{35}$ and COPD patients have a blunted Treg response to $\mathrm{CS}^{36}$

Adam 15 deficiency promotes pulmonary inflammation and emphysema development, in part, by reducing CS-induced activation of the mitochondrial apoptosis pathway in $\mathrm{CD}^{+}$ 
a

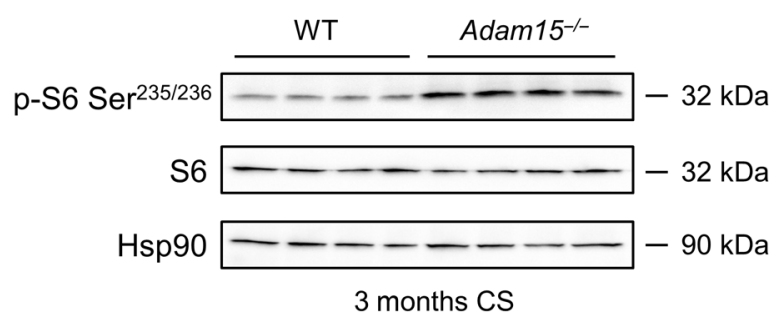

C

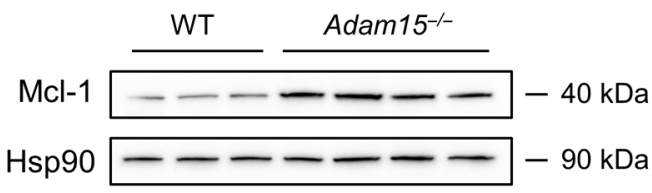

3 months CS b

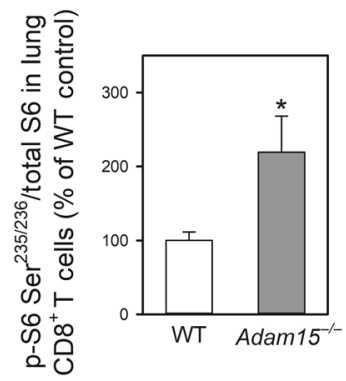

d

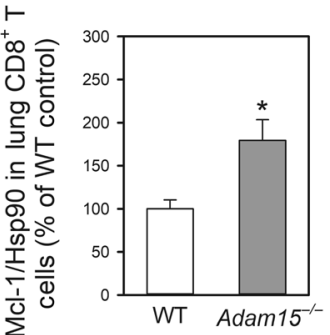

e

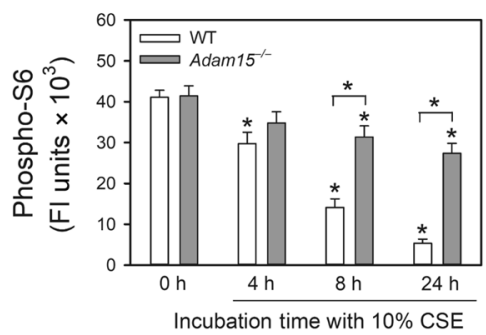

g

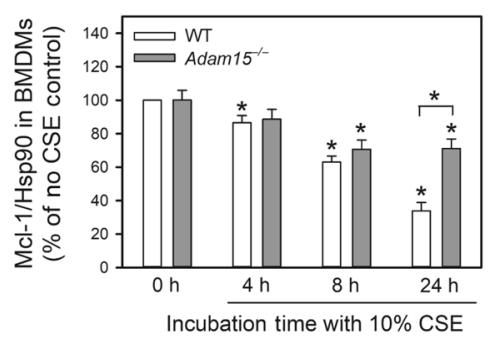

f WT Adam15 $1-$ CSE 0 h $4 \mathrm{~h} 8 \mathrm{~h} 24 \mathrm{~h} 0 \mathrm{~h} 4 \mathrm{~h} 8 \mathrm{~h} 24 \mathrm{~h}$

$\mathrm{Mcl}-1$

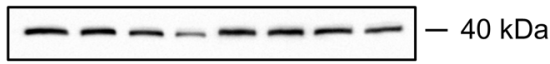

Hsp90 $-\longrightarrow--\longrightarrow-\longrightarrow-90 \mathrm{kDa}$

Fig. 8 Deficiency of Adam 15 in macrophages and CD8 ${ }^{+} \mathbf{T}$ cells preserves activation of the mTOR-Mcl-1 prosurvival pathway when cells are exposed to CS. a-d CD8 ${ }^{+}$T cells were isolated from the lungs of WT and Adam $15^{-/-}$mice that had been exposed to CS for 3 months. a Phosphorylated S6 (p-S6-Ser ${ }^{235 / 236}$ ), total S6 (S6), and a housekeeping control (heat shock protein-90; Hsp90) were quantified in extracts of the pulmonary $\mathrm{CD}^{+}{ }^{+} \mathrm{T}$ cells using Western blotting and densitometry. The $\mathrm{p}$-S6-Ser ${ }^{235 / 236}$ signals were normalized to total S6 levels and expressed as a \% of the values for WT mice. The images shown in a are representative of four mice per group. $\mathbf{b}$ The bars show means \pm SD ( $n=4$ mice/group). Data were analyzed using a one-way ANOVA followed by pair-wise testing with two-tailed Students $t$ tests. ${ }^{*} P<0.01$ versus

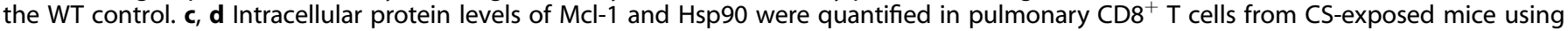
Western blotting and densitometry. The Mcl-1 signals were normalized to Hsp90 levels and expressed as a \% of the values for WT mice. The images shown in c are representative of 3-4 mice per group. $\mathbf{d}$ The bars show means \pm SD ( $n=3-4$ mice/group). Data were analyzed using a one-way ANOVA followed by pair-wise testing with two-tailed Students $t$ tests. ${ }^{*} P<0.004$ versus the WT control. e-g Bone marrow-derived macrophages (BMDMs) were isolated from unchallenged WT and Adam $15^{-1-}$ mice and exposed to $10 \%$ CSE for up to $24 \mathrm{~h}$. e BMDMs were fixed, permeabilized, and immunostained with a green fluorophore for intracellular phosphorylated ribosomal protein S6 (p-S6), and the nuclei were counterstained blue with 4',6-diamidino-2-phenylindole. Control cells were stained with a primary nonimmune rabbit (Rb) IgG. PS6 staining in BMDMs was quantified, as described in the "Methods". Data are mean \pm SD and data were analyzed using a one-way ANOVA followed by pair-wise testing with two-tailed Students $t$ tests. ${ }^{*} P<0.01$ versus the control. $\mathbf{f}, \mathbf{g}$ Intracellular protein levels of Mcl-1 and Hsp90 were quantified in CSE-treated BMDMs using Western blotting and densitometry. The images shown in $\mathbf{g}$ are representative of seven mice/ group. $g$ The Mcl-1 signals were normalized to Hsp90 levels and expressed as a \% of the values for controls cells incubated without CSE. Data are mean \pm SD; $n=7$ mice/group. Data were analyzed using a one-way ANOVA followed by pair-wise testing with two-tailed Student's $t$ tests. ${ }^{*} P \leq 0.004$ versus no CSE control belonging to the same genotype or the group indicated. 

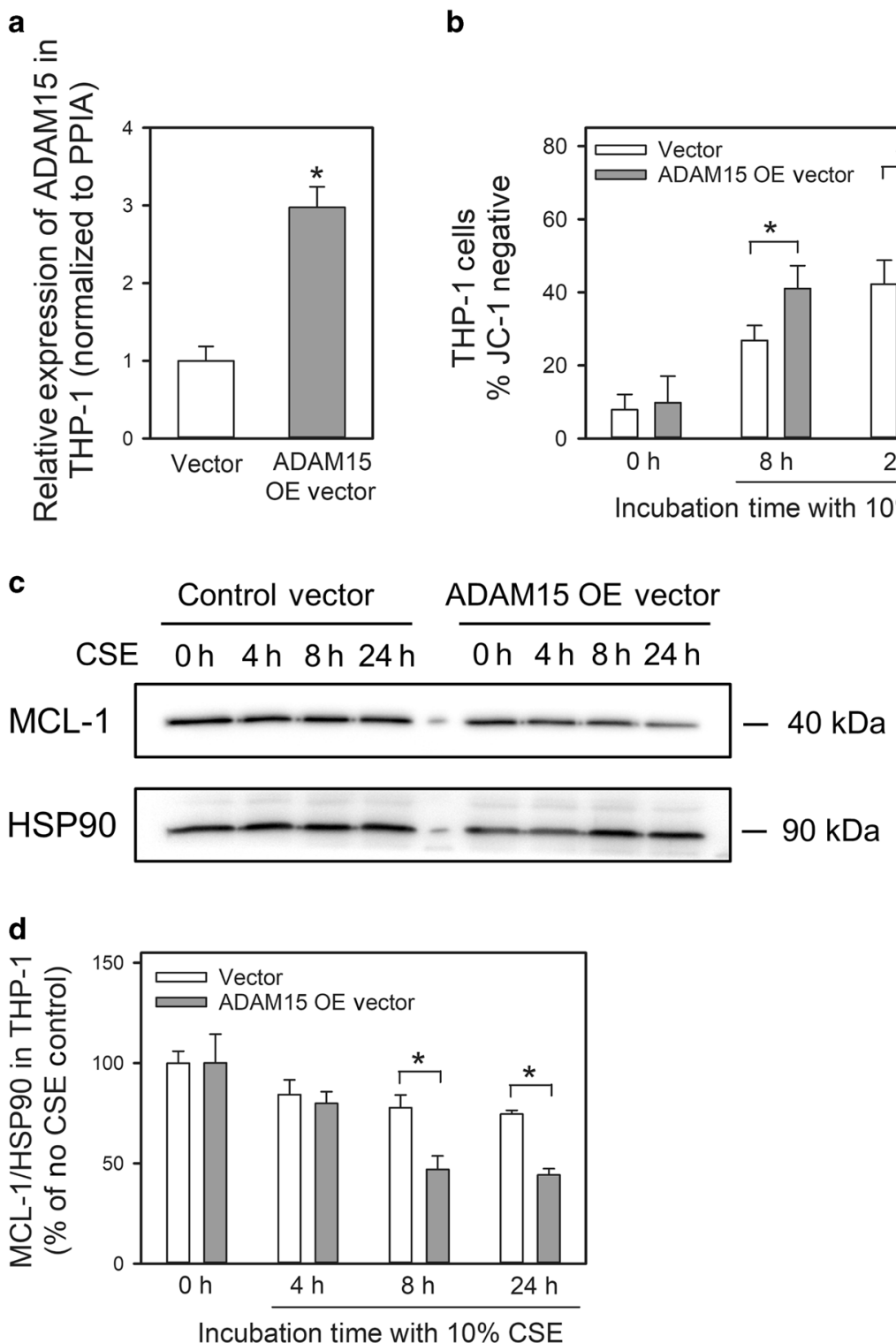

Fig. 9 Overexpression of ADAM15 in human THP-1 cells promotes CSE-induced apoptosis and reductions in intracellular Mcl-1 protein levels. a $A D A M 15$ steady state mRNA levels were measured in total cellular RNA samples from differentiated THP-1 $24 \mathrm{~h}$ after electroporation of an $A D A M 15$ over-expression [OE] vector (pcDNA3.1-ADAM15, a plasmid containing an ADAM15 complementary DNA) or a control vector (Vector) using quantitative real-time PCR. b-d Differentiated THP-1 cells were electroporated with either the ADAM15 OE vector or the control vector and then incubated with $10 \%$ CSE for the time indicated. b Loss of mitochondrial membrane potential was measured by staining the cells with JC-1 dye and quantifying the staining using MetaMorph software. c, d Intracellular protein levels of myeloid cell leukemia-1 (MCL-1) and heat shock protein-90 (HSP90; a housekeeping control) were quantified in CSE-treated THP-1 cells using Western blotting and densitometry. The MCL-1 signals were normalized to HSP90 levels and expressed as a \% of the values for controls cells incubated without CSE. Data are mean \pm SD. Data were analyzed using a one-way ANOVA followed by pair-wise testing with two-tailed Student's $t$ tests. ${ }^{*} P<0.03$ versus the group indicated.

T cells and macrophages thereby increasing their survival in the lung by abrogating CSE-induced reductions in mTOR signaling and downstream synthesis of $\mathrm{Mcl}-1$ protein. $\mathrm{Mcl}-1$ binds to and stabilizes the mitochondrial membrane thereby preventing CSinduced mitochondrial depolarization, cytochrome c release, and cellular apoptosis. Adam 15 deficiency in macrophages rendered these cells resistant to CSE-induced apoptosis in vitro. However, studies of CS-exposed Adam $15^{-1-} x C d 8^{-1-}$ mice demonstrated that Adam15 deficiency in $\mathrm{CD}^{+}{ }^{+} \mathrm{T}$ cells was required for the increased lung inflammation (including the increased lung macrophage counts) in CS-exposed Adam $15^{-1-}$ mice. CD8 ${ }^{+}$ $\mathrm{T}$ cells release $\mathrm{Cxcl} 10$ which activates mTOR signaling in other leukocytes by binding to Cxcr3 expressed on leukocytes and lung
Cxcl-10 levels were increased in CS-exposed Adam $15^{-1-}$ mice. ${ }^{37}$ We hypothesize that the increased numbers of apoptosis-resistant $\mathrm{CD}^{+} \mathrm{T}$ cells in the lungs of CS-exposed Adam $15^{-1-}$ mice release increased quantities of ligands for $\mathrm{Cxcr} 3$ expressed by macrophages which, in turn, drives increased mTOR signaling (and survival) of macrophages in the lungs of Adam $15^{-1-}$ mice (Fig. 10). The mechanism by which Adam 15 deficiency maintains mTOR signaling in CS-exposed $\mathrm{CD}^{+}{ }^{+}$cells and macrophages is unclear, but Adam15 has a cytoplasmic tail which binds to intracellular proteins including Src kinases which regulate the PI3 kinase-Akt pathway upstream of mTOR signaling. ${ }^{38}$

Adam 15 deficiency may also increase macrophage counts in CSexposed lungs by increasing lung levels of mediators that 


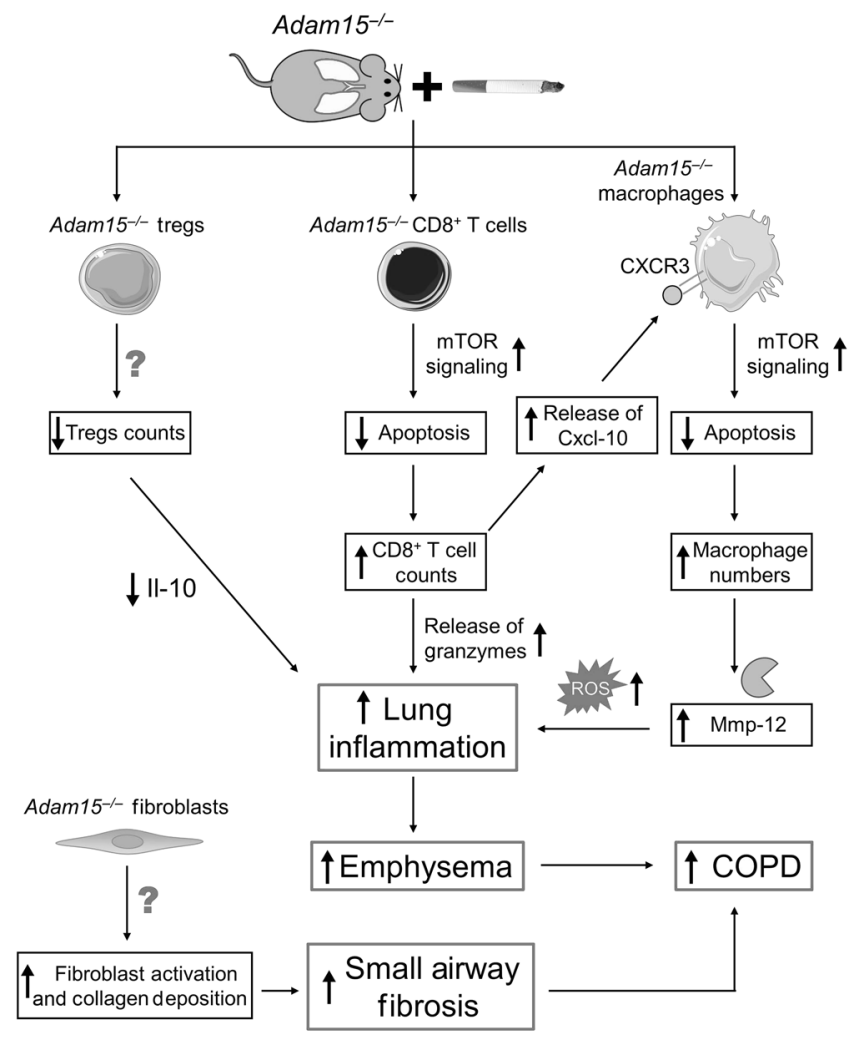

promote macrophage recruitment ( $\mathrm{Cl}-2)$ or survival (Gm-csf), or by decreasing lung levels of anti-inflammatory mediators including II-10. The reduced lung II-10 levels in CS-exposed Adam $15^{-1-}$ mice may be due to their reduced pulmonary Treg counts as II-10 is a Treg product. ${ }^{35}$ To our knowledge this is the first report linking deficiency of an Adam family member to Treg counts in any organ.

Adam 15 deficiency increased CS-induced small airway fibrosis and the numbers of a-SMA-positive cells (which could be myofibroblasts or smooth muscle cells) in the small airways of mice. Adam 15 expression was increased in small airway a-SMApositive cells in CS-exposed WT mice, and human ADAM15 increases human fibroblast migration and survival. ${ }^{14,39}$ Thus, Adam 15 deficiency in myofibroblasts may promote exaggerated small airway fibrosis in CS-exposed Adam $15^{-/-}$mice by increasing myofibroblast activation, survival, and/or ECM protein deposition. Although Tgf- $\beta$ promotes small airway fibrosis in mice, ${ }^{34} \mathrm{CS}$ exposed WT and Adam $15^{-1-}$ mice had similar lung Tgf- $\beta$ levels. The greater CS-induced small airway fibrosis in Adam $15^{-1-}$ mice was linked to their greater lung inflammation by BM transplant experiments. It is noteworthy that macrophage products promote small airway remodeling in CS-exposed rodents. ${ }^{40}$

A limitation of this study is that we employed only a loss-offunction approach in mice. Some of our findings could be due to compensatory changes in lung levels of other proteinases in Adam $15^{-1-}$ mice. Small molecules inhibitors which are selective for Adam15 do not exist. However, our ADAM15 overexpression results in THP-1 cells support our hypothesis that ADAM15 has protective activities in CS-exposed lungs by limiting the survival of leukocytes in the lung. Future studies will employ gain-of-function approaches in CS-exposed mice to test the hypothesis that Adam 15 has protective activities in the CS-exposed lung. We did not fully elucidate the mechanisms by which Adam 15 regulates small airway fibrosis or the contributions of PMN-derived Adam15 to CS-induced COPD-like disease which will be explored in future studies. Our in vitro studies evaluated the effects of CSE on
Fig. 10 Cartoon illustrating the mechanisms by which deficiency of Adam 15 leads to exaggerated COPD in mice. Exposing Adam $15^{-/}$ mice to CS leads to a phlogistic pulmonary response characterized by increases in the number of innate and adaptive immune cells (alveolar macrophages [AMs], $\mathrm{CD}^{+}$lymphocytes, $\mathrm{CD} 8^{+}$lymphocytes and CD3-positive NKT cells) and reductions in leukocytes having anti-inflammatory activities (Foxp ${ }^{+} \mathrm{T}$ regulatory cells) in the lung, along with exaggerated emphysema development and small airway fibrosis. The results of studies of Adam 15 bone marrow chimeras and Adam $15^{-1-} \mathrm{X} \mathrm{Cd8^{-1- }}$ mice indicate that deficiency of Adam 15 in leukocytes (especially $\mathrm{CD}^{+} \mathrm{T}$ cells) drives the increased emphysema development and small airway fibrosis observed in CSexposed Adam $15^{-1-}$ mice. Likely, increased release of Mmp-12 and oxidants from the increased numbers of macrophages and the increased release of granzymes from the increased numbers of $\mathrm{CD} 8^{+} \mathrm{T}$ cells detected in the lungs of CS-exposed Adam $15^{-/-}$mice contributed to their increased emphysema development. The exaggerated small airway fibrosis in CS-exposed Adam $15^{-/-}$mice may be due to: (1) increased release of mediators from leukocytes and other cells that activate (myo)fibroblasts; and (2) Adam15 deficiency in (myo)fibroblasts increasing the survival and/or profibrotic activities of these cells in the small airways. Adam15 deficiency increases CS-induced pulmonary inflammation by several mechanisms. First, Adam $15^{-1-} \mathrm{CD}^{+} \mathrm{T}$ cells (and macrophages) are protected from CS-induced activation of the mitochondrial apoptosis pathway and consequently accumulate in, and injure, the lung. Adam15 deficiency preserves mammalian target of rapamycin (mTOR) signaling in $\mathrm{CD}^{+} \mathrm{T}$ cells (and macrophages), leading to preserved expression of myeloid cell leukemia sequence-1 (Mcl-1) protein (an anti-apoptotic protein which binds to the mitochondrial membrane to prevent loss of mitochondrial membrane potential, mitochondrial release of cytochrome $c$ and downstream caspase activation). The increased number of $\mathrm{CD}^{+}{ }^{+} \mathrm{T}$ cells in lungs of Adam $15^{-1-}$ mice also promotes the survival of macrophages in the lung via Adam $15^{-1-} \mathrm{CD}^{+} \mathrm{T}$ cells releasing increased quantities of Cxcl-10 (and possibly other ligands for $\mathrm{Cxcr3}$ ), which bind to $\mathrm{Cxcr3}$

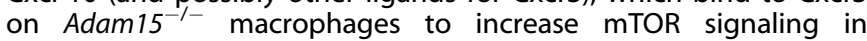
macrophages leading to increased macrophage survival and activation and release of $\mathrm{Mmp}-12$, oxidants and other mediators that promote lung inflammation and destruction. Second, the accumulation of leukocytes leads to increased release of proinflammatory mediators that amplify pulmonary inflammation and destruction in Adam $15^{-1-}$ lungs. Third, lung levels of antiinflammatory mediators, such as interleukin-10 (II-10) are reduced in CS-exposed Adam $15^{-/-}$lung, which may be due to the reduced numbers of Foxp ${ }^{+}$T regulatory cells (which are a source of II-10) in their lungs. A reduced anti-inflammatory immune response likely contributes to the phlogistic environment that increases emphysema development and small airway fibrosis in CS-exposed Adam $15^{-1-}$ lungs.

leukocytes and epithelial cells using CSE concentrations that were optimized for each cell type and readout. These studies only provide information on the effects of aqueous-soluble CS components, and it is not possible to relate the CSE concentrations that were tested to concentrations of gaseous-phase CS which contains other noxious components.

Our results suggest that strategies that increase lung levels of ADAM15 have therapeutic potential in COPD. However, increased expression of ADAM15 has been detected in various tumors including non-small cell lung cancer and is linked to tumor cell migration and metastasis by increasing MMP-9 expression. ${ }^{41,42}$ Additional studies are needed to evaluate ADAM15 as a potential therapeutic target for COPD.

In conclusion, our results strongly link Adam 15 deficiency to the pathogenesis of COPD-like disease in mice and mTOR signaling in pulmonary $\mathrm{CD}^{+} \mathrm{T}$ cells and macrophages in CS-exposed mice for the first time. In CS-exposed mice, Adam 15 deficiency leads to exaggerated COPD, in part, by increasing the pulmonary inflammatory response to $\mathrm{CS}$ by protecting $\mathrm{CD}^{+} \mathrm{T}$ cells and macrophages from CS-induced activation of the mitochondrial 
apoptosis pathway by preserving mTOR-Mcl-1 signaling in these leukocytes.

\section{METHODS}

Ethics

Studies conducted on mice were approved by the Institutional Care and Use Committee at Brigham and Women's Hospital (BWH). Studies conducted on human subjects were approved by the BWH Institutional Review Board.

\section{Mice}

Adam $15^{-1-}$ mice were obtained from Dr. CP Blobel (Cornell University, New York, NY) and backcrossed into a pure (57BL/ 6 strain (to the F8 generation). Adam $15^{-1-}$ mice have normal lung development and no abnormal phenotype in the unchallenged state. ${ }^{18}$ Pure strain $\mathrm{C} 57 \mathrm{BL} / 6 \mathrm{WT}$ mice and $\mathrm{Cd} 8^{-/-}$mice were obtained from the Jackson Laboratory (Bar Harbor, ME). Adam $15^{-1-}$ $x \mathrm{Cd} 8^{-/-}$mice were generated by breeding Adam $15^{-/-}$mice and $C d 8^{-/-}$mice. Genotypes were confirmed using PCR methods performed on DNA extracted from tail biopsies. Mice were housed under specific pathogen-free conditions until they were 8-12 weeks of age before experiments were conducted. Male and female were studied and randomized to the experimental groups by an observer not involved in the conduct of the experiments.

Adam 15 BM chimeric mice

Unchallenged C57BL/6 WT and Adam $15^{-1-}$ recipient mice aged 10-12 weeks were lethally-irradiated (using 450 centigray dose from a ${ }^{137}$ Cesium source ${ }^{8,43,44}$ twice $4 \mathrm{~h}$ apart) and then received complete BM (erythrocytes and leukocytes) containing two million leukocytes from non-irradiated donor mice via a tail vein injection. Four groups of Adam15 BM chimeras were generated by transplanting: (1) WT BM into WT recipients; (2) WT BM into Adam $15^{-1-}$ recipients; (3) Adam $15^{-1-}$ BM into WT recipients; and (4) Adam $15^{-/-}$BM into Adam $15^{-1-}$ recipients. Mice were house for 10 weeks to permit engraftment of donor BM and then 6 month air and CS exposures were initiated and lungs were removed (at $\sim 46$ weeks of age). ${ }^{8}$

\section{CS exposures}

Mice were exposed to air or mixed mainstream and side-stream CS from 3R4F Kentucky Research cigarettes for 2 h/day 6 days-aweek in Teague TE $10 \mathrm{z}$ chambers for $1-6$ months. ${ }^{45}$

\section{CS extract}

Smoke from two 3R4F cigarettes was bubbled through $20 \mathrm{ml}$ of DMEM medium yielding a $100 \%$ CSE solution which was filter sterilized $(0.2 \mu \mathrm{m}$ syringe filter), diluted, and used within $5 \mathrm{~min}$ of preparation. ${ }^{7,8}$ Pilot experiments optimized the CSE concentrations needed to induce Adam 15 expression or apoptosis for each cell type studied.

Adam 15 protein and mRNA levels

Adam 15 protein levels were measured in homogenates of WT lungs using an ELISA (CUSABIO, College Park, MD) and normalized to total protein levels measured in the same samples using a kit (Thermo-Fisher Scientific, Rockford, IL). Adam 15 mRNA levels were measured using a SYBR green-based real-time PCR assay with Ppia as the housekeeping gene (Table E2) and the comparative threshold method.

Immunostaining of $\mathrm{AMs}$ and $\mathrm{CD}^{+}{ }^{+} \mathrm{T}$ cells for surface Adam 15 AMs were isolated from murine lungs using BAL. Macrophages were isolated from the peritoneal cavities of mice 4 days after intraperitoneal injection of $40 \mathrm{mg}$ of thioglycollate in $1 \mathrm{ml}$ of DMEM medium using peritoneal lavage. Macrophages were cultured in DMEM containing $10 \%$ heat-inactivated fetal bovine serum (FBS), $100 \mathrm{U} / \mathrm{ml}$ penicillin and $100 \mu \mathrm{g} / \mathrm{ml}$ streptomycin for $48 \mathrm{~h}$ in 8 well Lab-Tek ${ }^{\oplus}$ Chamber slides ${ }^{\mathrm{Tm}}$ to render them quiescent. Macrophages were then incubated at $37^{\circ} \mathrm{C}$ for $8-48 \mathrm{~h}$ with $0-5 \%$ CSE.

$\mathrm{CD}^{+}$lymphocytes were isolated from the spleens of unchallenged mice using EasySep ${ }^{\mathrm{Tm}}$ Mouse $\mathrm{CD}^{+}{ }^{+} \mathrm{T}$ Cell Isolation Kits (STEMCELL Technologies, Cambridge, MA). CD8 ${ }^{+} \mathrm{T}$ cells were incubated at $37^{\circ} \mathrm{C}$ for up to $2 \mathrm{~h}$ with $0-2.5 \%$ CSE. Cells were fixed for $3 \mathrm{~min}$ at $4{ }^{\circ} \mathrm{C}$ in $3 \%$ paraformaldehyde and $0.25 \%$ glutaraldehyde in PBS, and immunostained for surface Adam15 using rat anti-Adam15 lgG or non-immune rat IgG followed by Alexa-488conjugated goat anti-rat $\mathrm{F}(\mathrm{ab})_{2}$. Cell surface immunofluorescence was quantified on images of the cells using MetaMorph software (Molecular Devices LLC, Sunnyvale, CA)., ${ }^{76}$

\section{ADAM15 mRNA levels in HBECs}

Normal HBECs (Lonza, Allendale, NJ) were incubated for 4-24 h with or without $2.5 \%$ CSE. Adam 15 mRNA levels were measured using quantitative real-time-PCR using PPIA as the housekeeping gene (Table E2).

Immunostaining of human leukocytes for surface ADAM15 Monocytes and $\mathrm{CD}^{+} \mathrm{T}$ cells were isolated from the blood of healthy donors by positive section for CD14 or CD8a using immunomagnetic beads (STEMCELL Technologies). Monocytes and CD8 ${ }^{+} \mathrm{T}$ cells were incubated with and without CSE and then immunostained for surface ADAM15, as described in the online supplement.

\section{COPD-like disease}

Mice were exposed to air or CS for 6 months, and serum samples were obtained post-euthanasia via right ventricular puncture. Lungs were inflated to $25 \mathrm{~cm} \mathrm{H}_{2} \mathrm{O}$ pressure and fixed in a $10 \%$ buffered formalin. Distal airspace size or fibrosis around small airways having a mean diameter of 300-699 microns was quantified on Gill's-stained or Masson's Trichrome-stained lung sections, respectively, using morphometric methods. ${ }^{7,8}$ Lung sections were immunostained for airway epithelial a-SMA (a myofibroblast or smooth muscle cell marker). ${ }^{7}$

BAL leukocyte counts

Total and differential leukocyte counts were performed on BAL samples. ${ }^{47}$

Lymphocyte subsets in enzymatic lung digests

Mice were exposed to air or CS for 1-3 months. Lung tissue was digested with $1 \mathrm{mg} / \mathrm{ml}$ collagenase D (Sigma Aldrich, St. Louis, MO) in medium at $37^{\circ} \mathrm{C}$ for $45 \mathrm{~min}$. The digests were passed through a $70 \mu \mathrm{m}$ strainer and washed with cold PBS containing $0.5 \%$ bovine serum albumin. The cells were fixed and permeabilized using the Foxp3 Staining Buffer Set (eBioscience, San Diego, CA). Cells were incubated with Fc block solution (Biolegend, San Diego, CA), and incubated with antibodies (Table E3) or isotype-matched control antibodies (rat IgG1k, IgG2ak, or IgG2bK) for $45 \mathrm{~min}$ at $4{ }^{\circ} \mathrm{C}$ (Table E3) and washed. After dead cell and doublet exclusion, lymphocyte subsets were identified as follows: (a) $\mathrm{CD}^{+} \mathrm{T}$ cells $\left(\mathrm{CD}_{4} 5^{+} \mathrm{CD}^{+} \mathrm{CD}^{+}\right)$, (b) T regulatory cells (Tregs; $\mathrm{CD} 45^{+} \mathrm{CD} 3^{+} \mathrm{CD} 4^{+}$ Foxp3 $\left.^{+}\right)$, (c) $\mathrm{CD}^{+}\left(\mathrm{CD}^{+} 5^{+} \mathrm{CD}^{+} \mathrm{CD}^{+} \mathrm{a}^{+}\right)$, (d) B cells $\left(\mathrm{CD} 45^{+} \mathrm{B} 22 \mathrm{O}^{+}\right)$, (e) CD3-negative NK cells $\left(\mathrm{CD} 45^{+} \mathrm{CD}^{-} \mathrm{Nk} 1.1^{+}\right.$), or (f) CD3-positive NKT cells $\left(\mathrm{CD}_{4} 5^{+} \mathrm{CD}^{+}{ }^{+} \mathrm{NK} 1.1^{+}\right)$. Cells were identified and quantified using flow cytometry with a BD LSR Fortessa Cell Analyzer (San Jose, CA) and counting beads (ThermoFisher, Waltham, MA). Data were analyzed with FlowJo software (Tree Star Inc., Palo Alto, CA).

\section{Lung mediator levels}

Mediators were measured in lung homogenates using ELISA kits and normalized to lung total protein levels measured on the aliquots of the same samples. ${ }^{7}$ 
Leukocyte apoptosis

AMs and splenic CD8 ${ }^{+} \mathrm{T}$ cells were isolated from unchallenged mice, as described above, and induced to undergo apoptosis by incubating them at $37^{\circ} \mathrm{C}$ with up to $10 \%$ CSE for up to $24 \mathrm{~h}$ at $37^{\circ}$ C. Cells were fixed (as described above), permeabilized with $100 \%$ methanol at $4{ }^{\circ} \mathrm{C}$ for $20 \mathrm{~min}$, and immunostained for intracellular active caspase-3 using Alexa-488-conjugated rabbit anti-caspase-3 IgG or Alexa-488-conjugated rabbit lgG as a control (Cell Signaling, Beverly, MA). The mean integrated fluorescence of 200 cells/group was quantified using MetaMorph software. Data were corrected for nonspecific staining in the presence of the nonimmune antibody. ${ }^{48}$ Other aliquots of cells were immediately stained for 30 min at $4{ }^{\circ} \mathrm{C}$ with JC- 1 dye using a Mitoprobe ${ }^{\circledR}$ kit (Thermo-Fisher Scientific, Rockford, IL). JC-1 staining in $>500$ cells/group was measured using MetaMorph software.

Ribosomal protein $\mathrm{S} 6$ phosphorylation in BM-derived macrophage (BMDMs)

BMDMs were isolated from naive mice, ${ }^{7,49}$ cultured in 8 -well chamber-slides in L929-conditioned medium for 7 days. The media were changed to complete DMEM medium (DMEM [Corning, Manassas, VA] supplemented with $10 \%$ heat-inactivated FBS [Sciencell Research Laboratories, Carlsbad, CA], 100 units/ml penicillin, and $100 \mu \mathrm{g} / \mathrm{mL}$ streptomycin [ThermoFisher Scientific, Waltham, MA]). Cells were incubated with or without $10 \%$ CSE and, at intervals, were fixed and incubated with rabbit anti-murine phospho-S6 (Ser ${ }^{235 / 236}$ ) lgG (Cell Signaling Technology, Beverly, MA), or non-immune rabbit lgG (Dako, Santa Clara, CA) followed by Alexa-488-conjugated goat anti-rabbit $\mathrm{F}(\mathrm{ab})_{2}$ (Invitrogen, Charlestown, MA). MetaMorph software was used to measure intracellular staining for phospho-S6.

Myeloid cell leukemia sequence-1 ( $\mathrm{Mcl}-1)$ protein levels in CSEtreated BMDMs

BMDMs were isolated from naïve mice, and then cultured to render them quiescent. ${ }^{7}$ Cells were incubated with or without $10 \%$ CSE and lyzed at intervals in radioimmunoprecipitation assay (RIPA) buffer containing proteinase inhibitors. Western Blot analysis for Mcl-1 and heat shock protein 90 (Hsp90; as a loading control) was performed and $\mathrm{Mcl}-1$ signals were normalized to Hsp90 signals. ${ }^{7}$

Ribosomal protein $\mathrm{S} 6$ phosphorylation and $\mathrm{Mcl}-1$ protein levels in pulmonary $\mathrm{CD}^{+} \mathrm{T}$ cells

Mice were exposed to air or to CS for 3 months. CD8 ${ }^{+} \mathrm{T}$ cells were isolated from enzymatic digests of lungs, as outlined above. Cells were immediately lyzed in RIPA buffer containing proteinase inhibitors and PhosSTOP (Sigma, St. Louis, MO). Western Blot analysis for $\mathrm{Mcl}-1$, phospho-S6 ( $\left.\mathrm{Ser}^{235 / 236}\right)$, total S6, and heat shock protein 90 ( $\mathrm{Hsp90}$; as a loading control) was performed. Mcl-1 signals were normalized to Hsp90 signals, phospho-S6 (Ser ${ }^{235 / 236}$ ) signals were normalized to total S6 signals.

Overexpression ADAM15 in human THP-1 cells

Human ADAM15 cDNA (isoform 6 preproprotein as the longest transcript variant) amplified by RT-PCR was subcloned into the BamHI and Xhol sites of pcDNA3.1 vector to obtain pcDNAADAM15 (the primer sequences used for cloning were: forward: $5^{\prime}-$ CGCGGTACCATGCGGCTGGCGCTGCTCTGGG-3'; reverse: 5' CCGCTCGAGTCAGAG GTAGAGCGAGGACACT-3'). Human THP-1 monocytes (from ATCC) were maintained in RPMI-1640 containing $10 \%$ FBS and differentiated into macrophage-like cells by incubating them with $150 \mathrm{nM}$ phorbol myristate acetate for $24 \mathrm{~h}$. Cells were electroporated (Neon Transfection System; Thermo Fisher Scientific, Waltham, MA) with the ADAM15 or control (pcDNA3.1) vector. $^{50}$ ADAM15 expression was measured using qRT-PCR and the THP-1 cells were treated with $10 \%$ CSE for up to
$24 \mathrm{~h}$. JC-1 staining and intracellular levels of MCL-1 were measured as described above.

Statistics

Data are presented as box-plots showing medians and $25^{\text {th }}$ and $75^{\text {th }}$ percentiles and whiskers showing $10^{\text {th }}$ and $90^{\text {th }}$ percentiles (for data that were not normally distributed) or mean \pm SD (or SEM; for data that were normally distributed). Data were analyzed with Kruskal-Wallis one-way ANOVA followed by pair-wise comparisons using Mann-Whitney $U$ tests (nonparametric data), or one-way ANOVAs followed by pair-wise comparisons using two-tailed Student's $t$ tests (parametric data). $P$ values $\leq 0.05$ were considered significant.

\section{ACKNOWLEDGEMENTS}

This work was supported by the following grants: Public Health Service, National Heart, Lung, and Blood Institute Grants HL063137, HL086814, HL111835, and Al111475-01, Flight Attendants Medical Research Institute grants \#CIA123046 and CIA052349, and The Department of Defense (CDMRP) grant \# PR152060 (to C.O.). Public Health Service, National Heart, Lung, and Blood Institute Grants F32HL147437 (to X.W.).

\section{AUTHOR CONTRIBUTIONS}

C.O. conceived the project and designed the experiments. X.W., D.Z., T.N., K.W., H.P., Y.L., Q.F., J.R., Y.T., and C.O. conducted the experiments or contributed to the data analysis. X.W. and C.O. contributed to the data interpretation and wrote the paper. All coauthors reviewed and approved the final paper.

\section{ADDITIONAL INFORMATION}

The online version of this article (https://doi.org/10.1038/s41385-020-0325-3) contains supplementary material, which is available to authorized users.

Competing interests: C.O. is an employee of Vertex Pharmaceuticals Inc. and may own stock in the company. The remaining authors have no competing interests.

Publisher's note Springer Nature remains neutral with regard to jurisdictional claims in published maps and institutional affiliations.

\section{REFERENCES}

1. Quaderi, S. A. \& Hurst, J. R. The unmet global burden of COPD. Glob. Health Epidemiol. Genom. 3, e4 (2018).

2. Owen, C. A. Roles for proteinases in the pathogenesis of chronic obstructive pulmonary disease. Int J. Chron. Obstruct. Pulmon. Dis. 3, 253-268 (2008).

3. Rabe, K. F. et al. Global strategy for the diagnosis, management, and prevention of chronic obstructive pulmonary disease: GOLD executive summary. Am. J. Respir. Crit. Care Med. 176, 532-555 (2007).

4. Kasahara, Y. et al. Inhibition of VEGF receptors causes lung cell apoptosis and emphysema. J. Clin. Invest 106, 1311-1319 (2000).

5. Shapiro, S. D. et al. Neutrophil elastase contributes to cigarette smoke-induced emphysema in mice. Am. J. Pathol. 163, 2329-2335 (2003).

6. Molet, S. et al. Increase in macrophage elastase (MMP-12) in lungs from patients with chronic obstructive pulmonary disease. Inflamm. Res. 54, 31-36 (2005).

7. Wang, X. et al. A Disintegrin and a metalloproteinase-9 (ADAM9): a novel proteinase culprit with multifarious contributions to COPD. Am. J. Respir. Crit. Care. Med. 198, 1500-1518 (2018).

8. Polverino, F. et al. A disintegrin and metalloproteinase domain-8: a novel protective proteinase in chronic obstructive pulmonary disease. Am. J. Respir. Crit. Care Med. 198, 1254-1267 (2018).

9. Owen, C. A. Leukocyte cell surface proteinases: regulation of expression, functions, and mechanisms of surface localization. Int J. Biochem. Cell Biol. 40, 1246-1272 (2008).

10. Dreymueller, D., Uhlig, S. \& Ludwig, A. ADAM-family metalloproteinases in lung inflammation: potential therapeutic targets. Am. J. Physiol. Lung Cell Mol. Physiol. 308, L325-L343 (2015).

11. Seals, D. F. \& Courtneidge, S. A. The ADAMs family of metalloproteases: multidomain proteins with multiple functions. Genes Dev. 17, 7-30 (2003). 
12. Martin, J., Eynstone, L. V., Davies, M., Williams, J. D. \& Steadman, R. The role of ADAM 15 in glomerular mesangial cell migration. J. Biol. Chem. 277, 33683-33689 (2002).

13. Maretzky, T. et al. Characterization of the catalytic activity of the membraneanchored metalloproteinase ADAM15 in cell-based assays. Biochem. J. 420, 105-113 (2009).

14. Herren, B. et al. ADAM15 overexpression in NIH3T3 cells enhances cell-cell interactions. Exp. Cell Res. 271, 152-160 (2001).

15. Maeno, T. et al. CD8+ T cells are required for inflammation and destruction in cigarette smoke-induced emphysema in mice. J. Immunol. 178, 8090-8096 (2007).

16. Grumelli, S. et al. An immune basis for lung parenchymal destruction in chronic obstructive pulmonary disease and emphysema. PLoS Med. 1, e8 (2004).

17. Hodge, S., Hodge, G., Nairn, J., Holmes, M. \& Reynolds, P. N. Increased airway granzyme $b$ and perforin in current and ex-smoking COPD subjects. COPD 3, 179-187 (2006).

18. Horiuchi, K. et al. Potential role for ADAM15 in pathological neovascularization in mice. Mol. Cell Biol. 23, 5614-5624 (2003).

19. Hiemstra, P. S. Altered macrophage function in chronic obstructive pulmonary disease. Ann. Am. Thorac. Soc. 10, S180-S185 (2013).

20. Marriott, H. M. et al. Dynamic changes in Mcl-1 expression regulate macrophage viability or commitment to apoptosis during bacterial clearance. J. Clin. Invest. 115, 359-368 (2005).

21. Bosque, A., Marzo, I., Naval, J. \& Anel, A. Apoptosis by IL-2 deprivation in human CD8 + T cell blasts predominates over death receptor ligation, requires Bim expression and is associated with Mcl-1 loss. Mol. Immunol. 44, 1446-1453 (2007).

22. Fulda, S. Synthetic lethality by co-targeting mitochondrial apoptosis and PI3K/ Akt/mTOR signaling. Mitochondrion 19(Pt A), 85-87 (2014).

23. Melgert, B. N. et al. Short-term smoke exposure attenuates ovalbumin-induced airway inflammation in allergic mice. Am. J. Respir. Cell Mol. Biol. 30, 880-885 (2004).

24. Khammas, H. et al. Characterisation of the human ADAM15 promoter. Nephron Exp. Nephrol. 118, e27-e38 (2011).

25. Mercer, B. A., Wallace, A. M., Brinckerhoff, C. E. \& D'Armiento, J. M. Identification of a cigarette smoke-responsive region in the distal MMP-1 promoter. Am. J. Respir. Cell Mol. Biol. 40, 4-12 (2009).

26. Aporntewan, C. et al. Gene hunting of the Genetic Analysis Workshop 16 rheumatoid arthritis data using rough set theory. BMC Proc. 3(Suppl 7), S126 (2009).

27. Osei, E. T. et al. Unravelling the complexity of COPD by microRNAs: it's a small world after all. Eur. Respir. J. 46, 807-818 (2015).

28. Chatterjee, V. et al. MicroRNA-147b regulates vascular endothelial barrier function by targeting ADAM15 expression. PLOS ONE 9, e110286 (2014).

29. Ryter, S. W., Alam, J. \& Choi, A. M. Heme oxygenase-1/carbon monoxide: from basic science to therapeutic applications. Physiol. Rev. 86, 583-650 (2006).

30. Liu, X., Wei, J., Peng, D. H., Layne, M. D. \& Yet, S. F. Absence of heme oxygenase-1 exacerbates myocardial ischemia/reperfusion injury in diabetic mice. Diabetes $\mathbf{5 4}$, 778-784 (2005)

31. Richards, J. A., Wigmore, S. J. \& Devey, L. R. Heme oxygenase system in hepatic ischemia-reperfusion injury. World J. Gastroenterol. 16, 6068-6078 (2010).

32. Sunderram, J. et al. Heme oxygenase-1-dependent central cardiorespiratory adaptations to chronic intermittent hypoxia in mice. J. Appl. Physiol. 121, 944-952 (2016).
33. Hautamaki, R. D., Kobayashi, D. K., Senior, R. M. \& Shapiro, S. D. Requirement for macrophage elastase for cigarette smoke-induced emphysema in mice. Science 277, 2002-2004 (1997).

34. Owen, C. A. Proteinases and oxidants as targets in the treatment of chronic obstructive pulmonary disease. Proc. Am. Thorac. Soc. 2, 373-385 (2005). discussion 394-375.

35. Schmitt, E. G. et al. IL-10 produced by induced regulatory T cells (iTregs) controls colitis and pathogenic ex-iTregs during immunotherapy. J. Immunol. 189, 5638-5648 (2012)

36. Barcelo, B. et al. Phenotypic characterisation of T-lymphocytes in COPD: abnormal CD4+CD25+ regulatory T-lymphocyte response to tobacco smoking. Eur. Respir. J. 31, 555-562 (2008).

37. Schwarz, J. B. et al. Novel role of the CXC chemokine receptor 3 in inflammatory response to arterial injury: involvement of mTORC1. Circ. Res. 104, 189-200 (2009).

38. Poghosyan, Z. et al. Phosphorylation-dependent interactions between ADAM15 cytoplasmic domain and Src family protein-tyrosine kinases. J. Biol. Chem. 277, 4999-5007 (2002).

39. Bohm, B. B., Freund, I., Krause, K., Kinne, R. W. \& Burkhardt, H. ADAM15 adds to apoptosis resistance of synovial fibroblasts by modulating focal adhesion kinase signaling. Arthritis Rheum. 65, 2826-2834 (2013).

40. Churg, A. et al. Effect of an MMP-9/MMP-12 inhibitor on smoke-induced emphysema and airway remodelling in guinea pigs. Thorax 62, 706-713 (2007).

41. Dong, D. D., Zhou, H. \& Li, G. ADAM15 targets MMP9 activity to promote lung cancer cell invasion. Oncol. Rep. 34, 2451-2460 (2015).

42. Fehringer, G. et al. Cross-cancer genome-wide analysis of lung, ovary, breast, prostate, and colorectal cancer reveals novel pleiotropic associations. Cancer Res. 76, 5103-5114 (2016).

43. Craig, V. J. et al. Profibrotic activities for matrix metalloproteinase-8 during bleomycin-mediated lung injury. J. Immunol. 190, 4283-4296 (2013).

44. Knolle, M. D. et al. Adam8 limits the development of allergic airway inflammation in mice. J. Immunol. 190, 6434-6449 (2013).

45. Laucho-Contreras, M. E., Taylor, K. L., Mahadeva, R., Boukedes, S. S. \& Owen, C. A. Automated measurement of pulmonary emphysema and small airway remodeling in cigarette smoke-exposed mice. J. Vis. Exp. https://doi.org/10.3791/52236 (2015).

46. Wang, X. et al. Tissue inhibitor of metalloproteinase-1 promotes polymorphonuclear neutrophil (PMN) pericellular proteolysis by anchoring matrix metalloproteinase-8 and -9 to PMN surfaces. J. Immunol. 202, 3267-3281 (2019).

47. Quintero, P. A., Knolle, M. D., Cala, L. F., Zhuang, Y. \& Owen, C. A. Matrix metalloproteinase-8 inactivates macrophage inflammatory protein-1 alpha to reduce acute lung inflammation and injury in mice. J. Immunol. 184, 1575-1588 (2010).

48. Owen, C. A, Hu, Z, Lopez-Otin, C. \& Shapiro, S. D. Membrane-bound matrix metalloproteinase- 8 on activated polymorphonuclear cells is a potent, tissue inhibitor of metalloproteinase-resistant collagenase and serpinase. J. Immunol. 172, 7791-7803 (2004).

49. Lee, H., Zhang, D., Zhu, Z., Dela Cruz, C. S. \& Jin, Y. Epithelial cell-derived microvesicles activate macrophages and promote inflammation via microvesiclecontaining microRNAs. Sci. Rep. 6, 35250 (2016).

50. Zhang, D. et al. A potential role of microvesicle-containing miR-223/142 in lung inflammation. Thorax 74, 865-874 (2019). 